\title{
CREB Signaling Is Involved in Rett Syndrome Pathogenesis
}

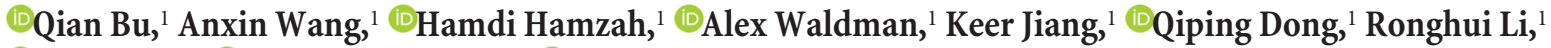 \\ (D)Jason Kim, ${ }^{1}$ DDaniel Turner, ${ }^{1}$ and ${ }^{\mathbb{D}}$ Qiang Chang ${ }^{1,2,3}$ \\ ${ }^{1}$ Waisman Center, ${ }^{2}$ Department of Medical Genetics, and ${ }^{3}$ Department of Neurology, University of Wisconsin-Madison, 1500 Highland Avenue, Madison, \\ Wisconsin 53705
}

Rett syndrome (RTT) is a debilitating neurodevelopmental disorder caused by mutations in the MECP2 gene. To facilitate the study of cellular mechanisms in human cells, we established several human stem cell lines: human embryonic stem cell (hESC) line carrying the common T158M mutation (MECP2 $\left.2^{T 158 M / T 158 M}\right)$, hESC line expressing no MECP2 (MECP2-KO), congenic pair of wild-type and mutant RTT patient-specific induced pluripotent stem cell (iPSC) line carrying the V247fs mutation (V247fs-WT and V247fs-MT), and iPSC line in which the V247fs mutation was corrected by CRISPR/Cas9-based genome editing (V247fs-MT-correction). Detailed analyses of forebrain neurons differentiated from these human stem cell lines revealed genotype-dependent quantitative phenotypes in neurite growth, dendritic complexity, and mitochondrial function. At the molecular level, we found a significant reduction in the level of CREB and phosphorylated CREB in forebrain neurons differentiated from MECP2 ${ }^{T 158 M / T 158 M}, M E C P 2-K O$, and V247fs-MT stem cell lines. Importantly, overexpression of CREB or pharmacological activation of CREB signaling in those forebrain neurons rescued the phenotypes in neurite growth, dendritic complexity, and mitochondrial function. Finally, pharmacological activation of CREB in the female Mecp2 heterozygous mice rescued several behavioral defects. Together, our study establishes a robust in vitro platform for consistent quantitative evaluation of genotype-dependent RTT phenotypes, reveals a previously unappreciated role of CREB signaling in RTT pathogenesis, and identifies a potential therapeutic target for RTT.

Key words: CREB; hESC; iPSC; MECP2; Rett syndrome; rolipram

Significance Statement

Our study establishes a robust human stem cell-based platform for consistent quantitative evaluation of genotype-dependent Rett syndrome (RTT) phenotypes at the cellular level. By providing the first evidence that enhancing cAMP response element binding protein signaling can alleviate RTT phenotypes both in vitro and in vivo, we reveal a previously unappreciated role of cAMP response element binding protein signaling in RTT pathogenesis, and identify a potential therapeutic target for RTT.

\section{Introduction}

Rett syndrome (RTT) is a debilitating neurodevelopmental disorder (Hagberg, 1985) caused by mutations in the X-linked methyl-CpG binding protein 2 (MECP2) gene (Amir et al., 1999). $\mathrm{MeCP} 2$ was initially characterized as a methyl-DNA binding protein (Lewis et al., 1992) and a suppressor of gene transcription (Nan et al., 1997). Consistent with this notion, the majority of RTT-causing mutations are found in either the methyl-CpG

Received Dec. 5, 2016; revised Feb. 17, 2017; accepted Feb. $24,2017$.

Author contributions: Q.B., A. Wang, Q.D., and Q.C. designed research; Q.B., A. Wang, H.H., A. Waldman, K.J., Q.D., R.L., J.K., and D.T. performed research; Q.B., A. Wang, H.H., A. Waldman, K.J., Q.D., R.L., and Q.C. analyzed data; Q.B. and Q.C. wrote the paper.

This work was supported in part by National Institutes of Health National Institute of Child Health and Human Development P30HD03352/U54HD090256 to the Waisman Center. We thank Xiaoji Zhang for technical support.

The authors declare no competing financial interests.

Correspondence should be addressed to Dr. Qiang Chang, University of Wisconsin-Madison, 1500 Highland Avenue, Madison, WI 53705. E-mail: qchang@waisman.wisc.edu.

DOI:10.1523/JNEUROSCI.3735-16.2017

Copyright $\odot 2017$ the authors $\quad 0270-6474 / 17 / 373671-15 \$ 15.00 / 0$ binding domain or the transcription repression domain (Samaco and Neul, 2011). Examples include the T158M missense mutation in methyl-CpG binding domain and the V247fs frame shift mutation in transcription repression domain. More recent work suggests that $\mathrm{MeCP} 2$ is also involved in transcriptional activation through recruiting the cAMP response element binding protein (CREB) to gene promoters (Chahrour et al., 2008). CREB is known to directly regulate the expression of specific genes (e.g., c-Fos, Arc, BDNF, and Wnt2) that underlie dendritic morphology (Wayman et al., 2006; Jan and Jan, 2010) and mitochondrial function (Merrill et al., 2011); and to indirectly regulate MeCP2 (Klein et al., 2007). Although defects in dendritic morphology and mitochondrial function have been observed in patient biopsies and RTT models (Eeg-Olofsson et al., 1990; Coker and Melnyk, 1991; Dotti et al., 1993; Cornford et al., 1994; Armstrong et al., 1995; Kishi and Macklis, 2004; Belichenko et al., 2009; Grosser et al., 2012; Li et al., 2013; Gold et al., 2014), the role of CREB in RTT pathogenesis has not been directly investigated. 
Following the identification of $M E C P 2$ as the disease gene, mouse models of engineered Mecp2 deletion were generated to study disease mechanisms (Chen et al., 2001; Guy et al., 2001; Shahbazian et al., 2002). Subsequently, RTT patient-specific induced pluripotent stem cell (iPSC) lines (Marchetto et al., 2010; Ananiev et al., 2011; Cheung et al., 2011; Kim et al., 2011) and human embryonic stem cell (hESC) line lacking MECP2 (Li et al., 2013) were generated to complement the mouse models. More recently, mouse lines carrying RTT disease-causing Mecp2 point mutations also became available (Goffin et al., 2011; Schaevitz et al., 2013; Wegener et al., 2014; Pitcher et al., 2015; Brown et al., 2016). While they offer better accessibility for studying molecular and cellular mechanisms, the hESCs, iPSCs, and their derivatives display highly variable biological characteristics among lines generated from different individuals due to differences in genetic background, thereby complicating their use in disease modeling (Soldner and Jaenisch, 2012). To establish a robust in vitro platform that can consistently produce quantitative genotypedependent RTT disease phenotypes at the cellular level, we created several hESC lines through the use of clustered-regularly interspaced-short-palindromic-repeats (CRISPR) based genome editing (Cong et al., 2013; Mali et al., 2013). These include hESC line carrying the common T158M mutation $\left(M E C P 2^{T 158 M / T 158 M}\right)$, hESC line expressing no MECP2 (MECP2$K O)$, and iPSC line in which the V247fs mutation was corrected (V247fs-MT-correction). Using forebrain neurons differentiated from these new hESC and iPSC lines, the congenic pair of wildtype (WT) and mutant RTT iPSC lines carrying the V247fs mutation (V247fs-WT and V247fs-MT) characterized in our previous studies (Ananiev et al., 2011; Williams et al., 2014), and the widely used parental hESC H9 line, we performed detailed quantification of characteristic RTT disease phenotypes in neurite growth and mitochondrial health. We found that both mutation in and loss of MECP2 dramatically impaired neurite growth, dendritic arborization, and mitochondrial function. In our search to identify molecular changes that connect MECP2 dysfunction with these cellular phenotypes, we discovered neurons differentiated from MECP2 mutant ESCs and iPSCs showed a significant reduction in CREB and phosphor-CREB levels. Furthermore, both overexpression of CREB and treatment with rolipram (pharmacological activator of CREB signaling) in mutant neurons significantly ameliorated the above-described cellular phenotypes. Finally, chronic administration of rolipram rescued several behavioral defects in female RTT mice. Together, our study establishes a robust in vitro platform for consistent quantitative evaluation of genotype-dependent RTT phenotypes, reveals a previously unappreciated role of CREB signaling in RTT pathogenesis, and identifies a potential therapeutic target for RTT.

\section{Materials and Methods}

DNA constructs. Human codon-optimized Cas9 nuclease and gRNAcloning vector were obtained from Addgene (plasmid \#44719 and plasmid \#41824) (Mali et al., 2013). The guide sequence of individual gRNAs was designed to specifically span junction sequences between the $5^{\prime}$ homology arm and $3^{\prime}$ homology arm, which only exists in the endogenous MECP2 allele (see Fig. 1). For correction of the V247fs mutation, the donor plasmid vector contained 5' and 3' arms of endogenous MECP2 sequence (obtained by PCR amplification of hESCs genomic DNA), the PGK promoter, and the puromycin resistance gene flanked by loxP sites. For engineering the T158M mutation, the donor plasmid was identical, except for replacement of threonine 158 with methionine in the $3^{\prime}$ arm. CREB or WNT2 cDNAs were subcloned into the Lox-Syn lentivirus vector (Gascón et al., 2008) to overexpress CREB or WNT2 in forebrain
Table 1. The primer information about the selected off-target sites

\begin{tabular}{lll}
\hline Name & Forward & Reverse \\
\hline gRNA77-1 & CATTATCTTTCTCTGACACCAG & TGAATAGACAGACACTGGAGG \\
gRNA77-2 & TTCACACCATCCTGTTGACATT & TTGGACCAGCTCCTCAGAA \\
gRNA77-3 & GTTCCTCCACCTTGTTCCAG & AGCATTCAGATTCCAACACAGT \\
gRNA77-4 & GGAGCTGTTGTTAAGGCATCA & TCTATCCACAAGAAGTGACCTG \\
gRNA77-5 & TTGTAGCGACGAAGGTGG & CTGGACTGCCTGTGTTGAA \\
gRNA53-1 & CTAAGTGTCAGGCAGGATC & CAGAATGAGCGTGAAGGT \\
gRNA53-2 & AGGAGGACACAGAATGGAT & CCTAGTTCTCACCAATCACT \\
gRNA53-3 & CTGGTCTTCCACACTCCT & CCTCCACTTGCCATTACTC \\
gRNA53-4 & TCTACTTGACTTCCACCTTC & CACGAGTGAATGAATGAACA \\
gRNA53-5 & CTCATAGGTCAGGCAGAAC & AGACGACGTGGAGTTGTA \\
\hline
\end{tabular}

neurons. This vector contains two neuron-specific synapsin promoters: one controls the expression of CREB or WNT2; and the other controls the expression of GFP or mitoDsRed2.

Cell culture. hESC line H9 was obtained from WiCell Research Institute (RRID:SCR_004364; www.wicell.org) and maintained on irradiated mouse embryonic fibroblast (MEF) feeders in hESC medium containing DMEM/F12 (Invitrogen), 20\% knock-out serum replacement (Invitrogen), $1 \times$ nonessential amino acids (Invitrogen), $0.5 \times$ GlutaMAX (Invitrogen), and $0.1 \mathrm{~mm} \beta$-mercaptoethanol (Sigma) and $4 \mathrm{ng} / \mathrm{ml} \mathrm{bFGF}$ (R\&D Systems). RTT iPSC lines were maintained the same way as the hESC lines.

Primary mouse astrocytes were isolated from the forebrain of newborn C57BL/6 mice. Briefly, newborn mouse forebrain was dissociated by harsh trituration to avoid growing of neurons and plated onto $10 \mathrm{~cm}$ dish in DMEM supplemented with 10\% FBS. Upon reaching confluence, glial cells were trypsinized and replated at a lower density for a total of 3 times to remove potential trace amounts of mouse neurons before the primary astrocyte cultures were used for coculture experiment with human neurons.

Generation of MECP2 mutant hESC lines and RTT iPSC mutationcorrection lines. Genome editing was performed as previously described (Chen et al., 2015). Briefly, hESCs or iPSCs were digested by TrypLE Enzyme for $3 \mathrm{~min}$ and dispersed into single cells in suspension. A total of $1 \times 10^{7}$ cells were electroporated with $15 \mu \mathrm{g}$ Cas 9 plasmid, $15 \mu \mathrm{g}$ gRNA plasmid, and 30 $\mu \mathrm{g}$ donor plasmid in $500 \mu \mathrm{l}$ of cold hESC medium using the Gene Pulser Xcell System at $250 \mathrm{~V}, 500 \mu \mathrm{F}$ in a $0.4 \mathrm{~cm}$ cuvette. Electroporated cells were subsequently plated onto three 6-well MEF-feeder plates in MEF-conditioned hESC medium with ROCK-inhibitor for $24 \mathrm{~h}$. Three days later, 0.5 $\mu \mathrm{g} / \mathrm{ml}$ puromycin was added for selection for $\sim 2$ weeks. Positive colonies were identified by PCR screening and sequencing. The PGK-puromycin cassette was removed by Cre recombinase. The primers used for amplifying human MECP2 sequences for genotyping in ESC and iPSC lines are as follows: (1) for genotyping the MECP2 ${ }^{\text {T158M }}$ allele: MECP2-T158M forward, AGCACCGTACCTGAGTTCAA; MECP2-T158M reverse, CTTTTCACC TGCACACCCTC; (2) for genotyping the MECP2-KO allele: MECP2-KO forward, GAAGCTCCTTGTCAAGATG; MECP2-KO reverse: CACC GAAAACGGGAGTGTCCTCTC; and (3) for genotyping the MECP2 ${ }^{\mathrm{V} 247 \mathrm{fs}}$ allele: MECP2-V247fs forward, ACAGTCCCCAGGGAAAAGCCTTTC; MECP2-V247fs reverse, GCTTCACCACTTCCTTGACCTC.

Off-target analysis in hESC and iPSC lines. The potential off-target sites were selected according to online tools provided by Feng Zhang's laboratory (http://crispr.mit.edu/) (Hsu et al., 2013). Five potential off-target sites were sequenced for each sgRNA target site. The primer information about the selected off-target sites is listed in Table 1.

Karyotyping. All karyotyping analyses were performed by the cytogenetics laboratory at the WiCell Research Institute (Madison, WI).

Neural differentiation. Human forebrain neurons were differentiated from hESCs as previously described (Chambers et al., 2009; Du et al., 2015) with minor modifications. Briefly, hESCs or iPSCs were passaged onto irradiated MEF plates using dispase $(1 \mathrm{mg} / \mathrm{ml})$ and cultured in hESC medium for $7 \mathrm{~d}$ until $\sim 90 \%$ confluent. To induce neural differentiation, hESC medium was replaced with a chemically defined neural medium, including DMEM/F12, Neurobasal medium at 1:1, $1 \times \mathrm{N} 2$, $0.5 \times$ B27, $0.5 \times$ Glutamax, and $1 \times$ NEAA (all reagents from Invitro- 
gen). The $2 \mu \mathrm{M}$ DMH1 (Torcris Biosciences) and $2 \mu \mathrm{M} \mathrm{SB} 431542$ (Stemgent) were added in the medium. The culture medium was changed every day. hESCs maintained under this condition for $8 \mathrm{~d}$ were induced into neuroepithelial progenitors. The neuroepithelial progenitor cells were then dissociated with dispase $(1 \mathrm{mg} / \mathrm{ml})$ and lifted up in neuronal induction medium, including DMEM/F12, $1 \times \mathrm{N} 2$, and $1 \times \mathrm{NEAA}$ for $5 \mathrm{~d}$ differentiated into neuronal progenitors (NPs). To differentiate the NP cells into neurons, NPs were dissociated with TrypLE Enzyme into single cells and plated on Matrigel-coated plates or on astrocytes in neuronal differentiation medium, including Neurobasal medium, $1 \times \mathrm{N} 2,1 \times$ B27, $0.5 \times$ Glutamax, and $1 \times$ NEAA, with $0.1 \mu \mathrm{M}$ Compound E (Calbiochem), $0.5 \mu \mathrm{M}$ ROCK inhibitor, and $1 \mu \mathrm{M}$ AraC. For identifying disease phenotypes, neurons were cultured in neuronal differentiation medium without neurotrophic factors.

Quantification of neuronal morphology and mitochondrial morphology. Forebrain neurons cultured for $21 \mathrm{~d}$ were transfected with Synapsin-GFP or Synapsin-mitoDsRed2, using the calcium phosphate precipitation method to achieve low transfection efficiencies (lower transfection efficiency gave sufficient separation between transfected neurons to facilitate morphological analysis). On day 28 , the culture was fixed with $4 \%$ PFA for $30 \mathrm{~min}$ and washed with PBS 3 times. For neuronal morphology analysis, GFP-positive neurons were randomly chosen from three or more independent cultures and imaged on an A1RSi confocal microscope system (Nikon) with a $20 \times$ objective. Ten to 20 neurons per condition per culture were included in the analyses. Confocal images were analyzed using the Neurolucida (RRID:SCR_001775; MicroBrightField, http://www.mbfbioscience.com/) image analysis software. Data were extracted for Sholl analysis and total dendritic length of each GFPpositive neuron. The person performing Sholl analysis was blind to the genotypes and treatments of the samples. For mitochondrial morphology analysis, mitoDsRed2-positive neurons were imaged using an A1RSi confocal microscope system (Nikon) with a $60 \times$ oil objective. The aspect ratio of mitochondria in neurites was analyzed using ImageJ (RRID:SCR_003070) with mitochondrial morphology plug-in as previously described (Dagda et al., 2009).

Measurement of mitochondrial membrane potential. The mitochondrial membrane potential in neurons was determined by JC-10 Mitochondrial Membrane Potential Assay Kit (Abcam), following the manufacturer's protocol. Briefly, neurons were stained with JC-10 solution for $30 \mathrm{~min}$ at $37^{\circ} \mathrm{C}$. After adding buffer $\mathrm{B}$, neurons were imaged using an A1RSi confocal microscope system (Nikon) with a $20 \times$ objective. The mitochondrial membrane potential was assessed by the quantifying the ratio of the intensity of red fluorescence (emission wavelength $590 \mathrm{~nm}$ ) to green fluorescence (emission wavelength $520 \mathrm{~nm}$ ).

Western blot analysis. Cultured neurons from 6-well plate were washed with PBS once and lysed by RIPA lysis buffer, containing protease inhibitor mixture (Roche) and phosphatase inhibitor mixture (Roche), and sonicated $15 \mathrm{~min}$. Protein concentration was measured using BCA protein assay (Bio-Rad). The samples were separated by SDS-PAGE and then transferred onto nitrocellulose membrane (Whatman). The membrane was blocked by $5 \%$ fat-free milk for $1 \mathrm{~h}$ at room temperature and then incubated with appropriate primary antibody diluted in 3\% BSA solution at $4^{\circ} \mathrm{C}$ overnight. After incubation with DyLight dye-conjugated secondary antibodies (Thermo Fisher Scientific catalog \#35518 RRID: AB_614942; \#SA5-35571 RRID:AB_2556775;1:10,000) for $1 \mathrm{~h}$ at room temperature, blots were scanned by the Odyssey Western Detection system (LI-COR Biosciences), followed by quantification with ImageStudio software (LI-COR Biosciences). For immunodetection, the following antibodies were used for our analysis: rabbit anti-MECP2 (Cell Signaling Technology catalog \#3456S RRID:AB_2143849), rabbit anti-CREB (Millipore catalog \#06-863 RRID:AB_310268), rabbit anti-phosphor-CREB (Cell Signaling Technology catalog \#9198, also 9198S, 9198L RRID: AB_2561044), mouse anti-GAPDH (Millipore catalog \#AB2302 RRID: AB_10615768).

Immunostaining. The immunostaining experiments were performed as previously described (Zhong et al., 2012) with minor modifications. Briefly, cultured cells were fixed with $4 \%(\mathrm{w} / \mathrm{v})$ PFA in PBS for $30 \mathrm{~min}$ at room temperature, and washed 3 times with PBS (10 min per wash). Following permeablization with $1 \%$ Triton X-100 for $30 \mathrm{~min}$, cells were blocked with
3\% normal donkey serum, $0.25 \%$ Triton X-100 in PBS (blocking buffer) for $1 \mathrm{~h}$ at room temperature. Cells were incubated with primary antibodies overnight at $4^{\circ} \mathrm{C}$, washed 3 times with PBS (10 min per wash), and incubated with the corresponding secondary antibodies for $1 \mathrm{~h}$ at room temperature. Primary antibodies included rabbit anti-MECP2 (Cell Signaling Technology catalog \#3456S RRID:AB_2143849), mouse anti-Tuj1 (Promega catalog \#G7121 RRID:AB_430874), rabbit anti-CREB (Millipore catalog \#06-863 RRID:AB_310268), and rabbit anti-phosphor-CREB (Cell Signaling Technology catalog \#9198, also 9198S, 9198L RRID:AB_2561044). Secondary antibodies were conjugated with either AlexaFluor-488 or AlexaFluor-568 (Thermo Fisher Scientific catalog \#A-21206, also A21206 RRID: AB_2535792; Thermo Fisher Scientific catalog \#A10037 RRID: AB_2534013). Nuclei were counterstained with DAPI. Images were taken using an A1RSi confocal microscope system (Nikon) with $10 \times, 20 \times$, or $60 \times$ objectives.

RNA extraction and $q R T-P C R$. Total RNA extraction from cells was performed using TRIzol (Invitrogen). RNA was reverse transcribed into cDNA using qScript cDNA SuperMix (Quanta Biosciences). qPCR was performed on an ABI Step-One plus machine using SYBR Green qPCR Master Mix (Biotool). GAPDH was used as loading control. The $2^{-\triangle \mathrm{Ct}}$ method was used to calculate fold change. Primers are as follows: WNT2 forward, ACTCT CAGGACATGCTGGCT; WNT2 reverse, ACGAGGTCATTTTTCGT TGG; GAPDH forward, AATCCCATCACCATCTTCCA; GAPDH reverse, TGGACTCCACGACGTACTCA.

Rolipram administration and behavioral testing in mice. All protocols were approved by the Institutional Animal Care and Use Committee at the University of Wisconsin-Madison. All mice are kept in the C57BL/6 background. Female Mecp $2^{-/+}$mice of 4-6 months of age were randomly assigned into two groups: one receiving vehicle (DMSO), the other receiving rolipram. Female $M e c p 2^{+/+}$littermates treated with vehicle (DMSO) were included as WT controls. For each mouse assigned to the rolipram group, rolipram was administered once daily by intraperitoneal injection at a dose of $0.03 \mathrm{mg} / \mathrm{kg}$ for 8 weeks. After the treatment stopped, the open field test and the fear conditioning test were performed as described in our previous study (Li et al., 2011).

Statistical analysis. Comparisons between two groups were determined by unpaired Student's $t$ tests. Comparisons among multiple groups were determined by one-way or two-way ANOVA followed by Bonferroni's or Dunnett's post hoc test. All tests were considered to be significant if $p<0.05$. All statistical analyses were performed using the GraphPad Prism software.

\section{Results}

\section{Generation and characterization of a series of human stem cell lines with engineered alterations at the endogenous MECP2 locus}

A total of $8.9 \%$ of RTT patients carry the mutation of threonine 158 to methionine (T158M), which is located at the $\mathrm{C}$ terminus of the methyl-CpG binding domain of MECP2. The T158M mutation is predicted to disrupt the association between MECP2 and methylated DNA. Yet, no human stem cell model carrying this most common RTT mutation has been generated. To establish a hESC line carrying the T158M mutation in the endogenous MECP2 locus, we electroporated the widely used hESC line H9 cells with plasmid DNAs encoding, respectively, the Cas9 enzyme, the MECP2-T158M donor template that carries the PGKpuromycin selection cassette flanked by the $5^{\prime}$ and $3^{\prime}$ homology arms of the endogenous MECP2 genomic sequence with the engineered T158M mutation (MECP2-T158M Donor Plasmid), and an sgRNA specifically recognizing an intronic site between exon 3 and exon 4 of MECP2 (sgRNA77; Fig. 1A). After drug selection, PCR genotyping, and DNA sequencing (Fig. 1B), the corrected targeted hESC clones homozygous for the T158M mutation $\left(M E C P 2^{T 158 M / T 158 M}\right)$ were treated with Cre recombinase to remove the PGK-puromycin cassette (Fig. 1A). To establish an hESC line expressing no MECP2, we used an sgRNA specifically targeting exon4 of MECP2 (sgRNA53; Fig. 2A), and identified a 
A

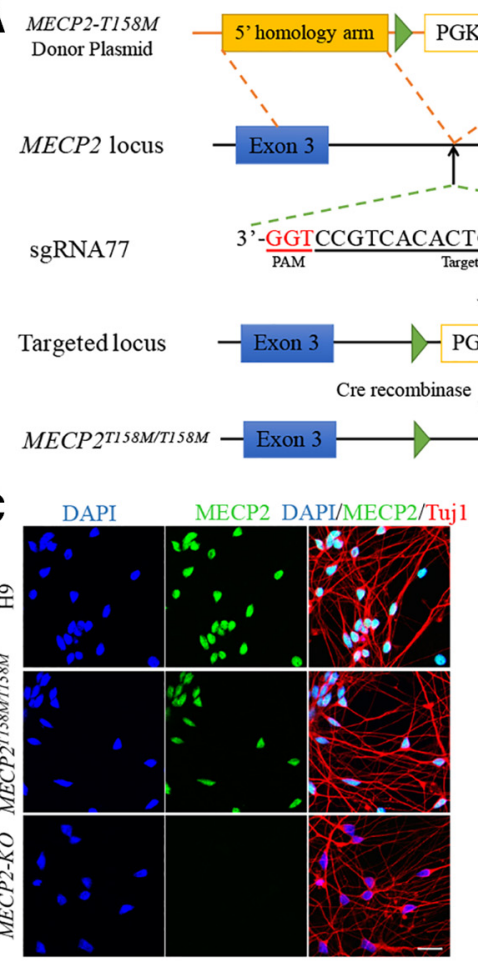

F

MECP2-WT Donor Plasmid

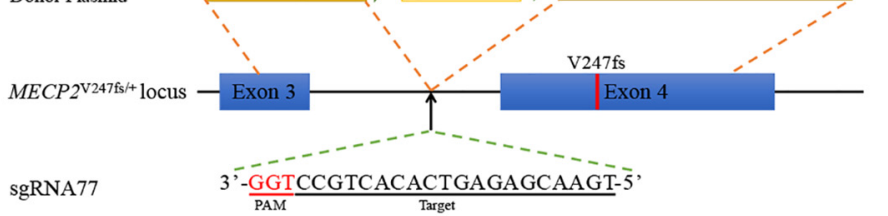

sgRNA77

Targeted locus

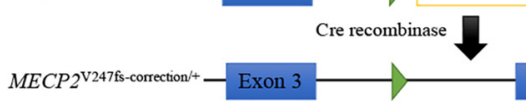

D ${ }_{100}$

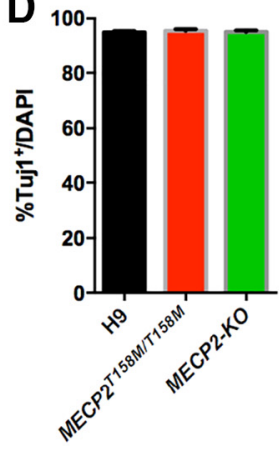

E

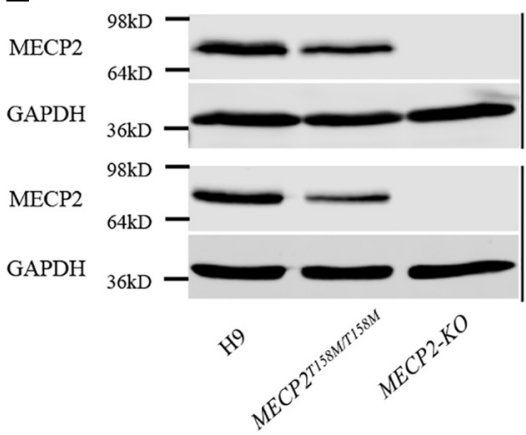

NPs

G

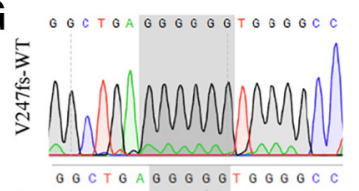

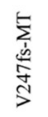

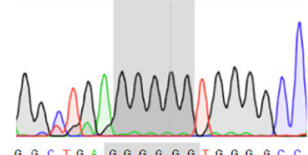

$\sum_{i}^{+}$.

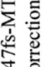

$\frac{D}{G A C T T C A C G T A A C T O G O}$

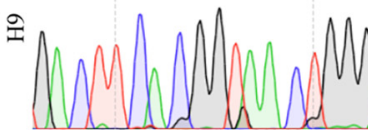

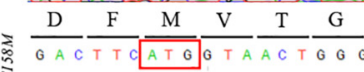

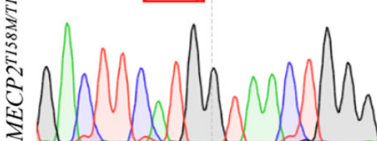

H DAPI MECP2 DAPI/MECP2/Tuj1 |
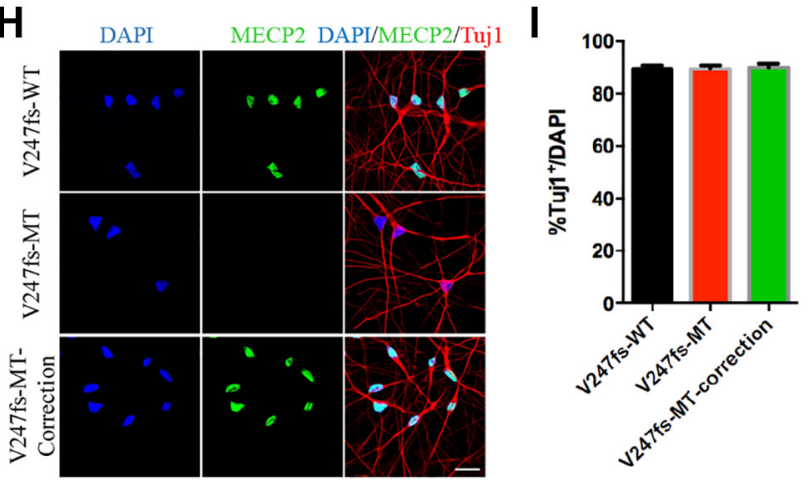

J
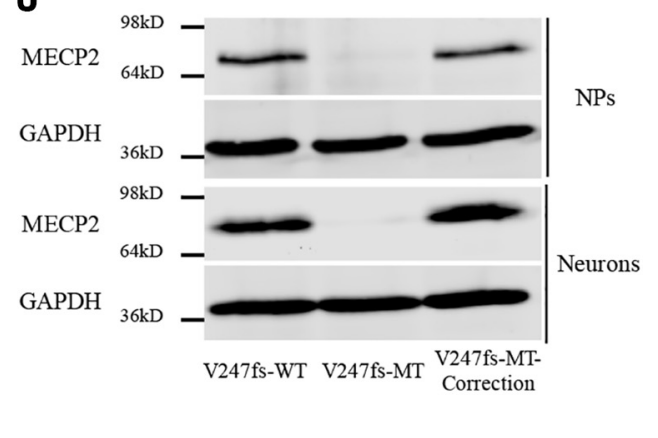

Figure 1. Generation and characterization of the MECP2 ${ }^{T 158 M / T 158 M} \mathrm{hESC}$ line and the V247fs-MT-correction iPSC line. A, Schematic diagram of the MECP2 ${ }^{T 158 M / 1158 M}$ targeting strategy in hESCS. Green triangles represent loxP sites. $B$, Representative sequencing chromatograms of DNA from the parental $H 9$ line and the MECP2 ${ }^{T 158 M / T 158 M}$ line. Single letter amino acid codes are on top of each sequencing chromatogram. Boxes represent the WT and mutant codon at amino acid 158. C, Representative fluorescence images of H9, MECP2 ${ }^{T 158 M / T 158 M}$, and MECP2-KO neurons COunterstained by DAPI (blue) and immunostained with antibodies specific against MECP2 (green) and Tuj1 (red). Scale bar, $20 \mu \mathrm{m}$. Scale bar applies to all images. D, Quantification of the Tuj ${ }^{+}$neuronal population as a percentage of all DAPI-labeled cells differentiated from the H9, MECP2 ${ }^{T 158 M / T 158 M}$, and MECP2-KO hESC lines. E, Western blot analyses of MECP2 in NPs and neurons differentiated from the H9, MECP2 ${ }^{T 158 M / T 158 M}$, and MECP2-KO hESC lines. $F$, Schematic diagram of the V247fs mutation correction strategy in RTT iPSCs. Green triangles represent loxP sites. $\mathbf{G}$, Representative sequencing chromatograms of RT-PCR products of MECP2 coding region containing the V247fs mutation from the V247fs-WT, V247fs-MT, and V247fs-MT-correction iPSC lines. H, Representative fluorescence images of V247fs-WT, V247fs-MT, and V247fs-MT-correction neurons counterstained by DAPI (blue) and immunostained with antibodies specific against MeCP2 (green) and Tuj1 (red). Scale bar, $20 \mu \mathrm{m}$. Scale bar applies to all images. I, Quantification of the Tuj ${ }^{+}$neuronal population as a percentage of all DAPI-labeled cells differentiated from the V247fs-WT, V247fs-MT, and V247fs-MT-correction neurons.J, Western blot analyses of MECP2 in NPs and neurons differentiated from V247fs-WT, V247fs-MT, and V247fs-MT-correction iPSC lines. Bar graphs, Data are mean \pm SEM. 


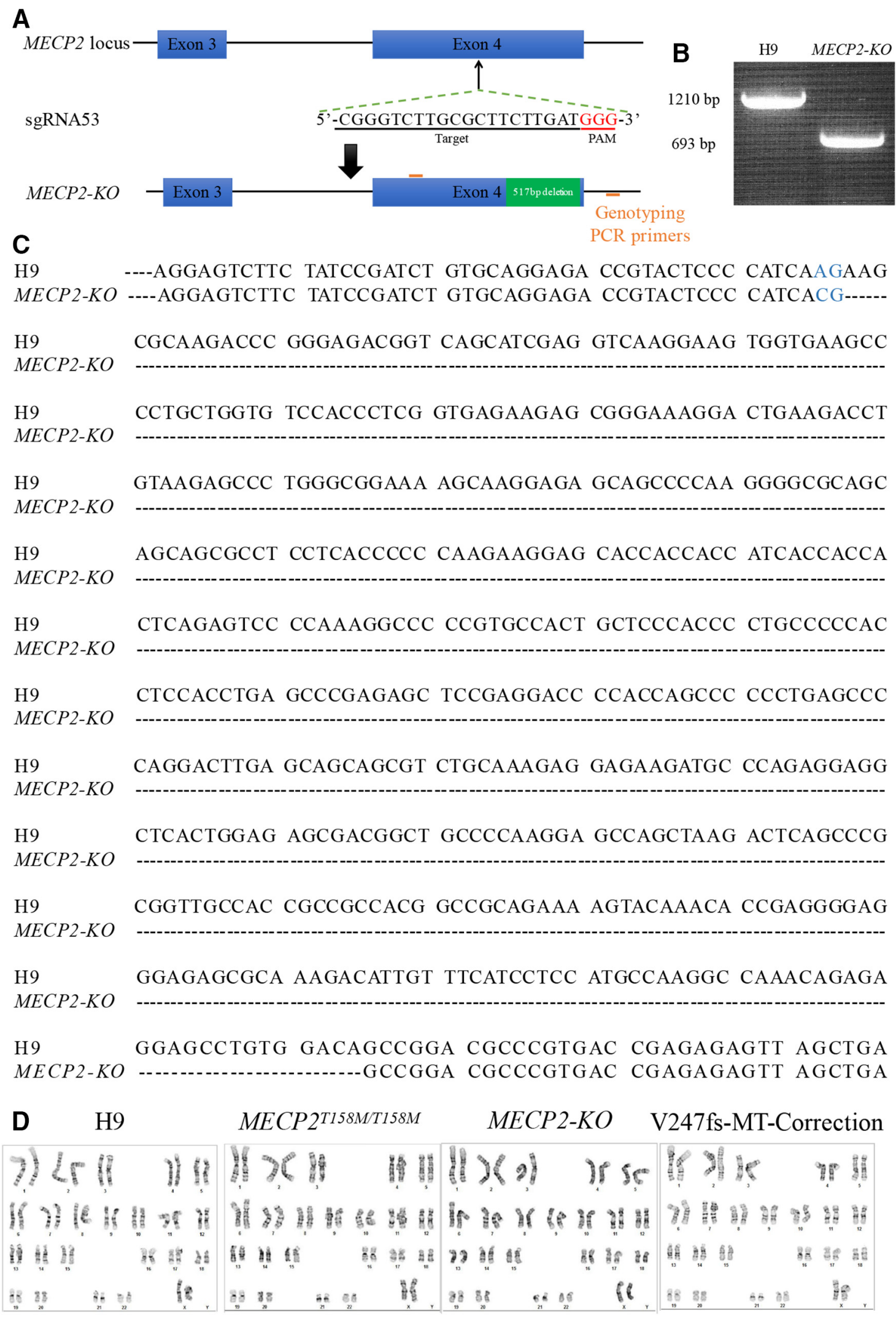

Figure 2. Generation and characterization of the MECP2-KO hESC line. A, Schematic diagram of the MECP2-KO targeting strategy in hESCS. B, PCR analysis of H9 and MECP2-KO hESC clones using primers shown in $A$. C, Representative sequencing chromatograms of DNA from the H9 and MECP2-KO hESC lines, showing the 517 bp deletion in the MECP2-KO line. $D$, Cytogenetic analysis of the H9, MECP2 ${ }^{T 158 M / T 158 M}$, and MECP2-KO hESC lines and the V247fs-MT-correction iPSC line, showing a normal karyotype in each line. 
A

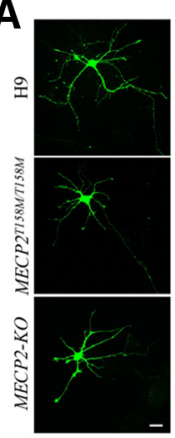

D

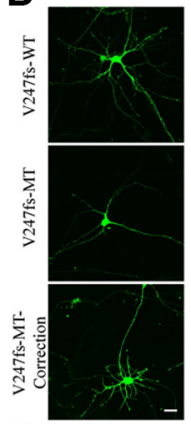

G
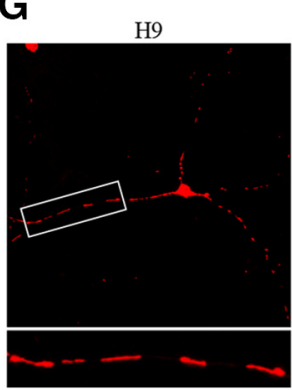

I
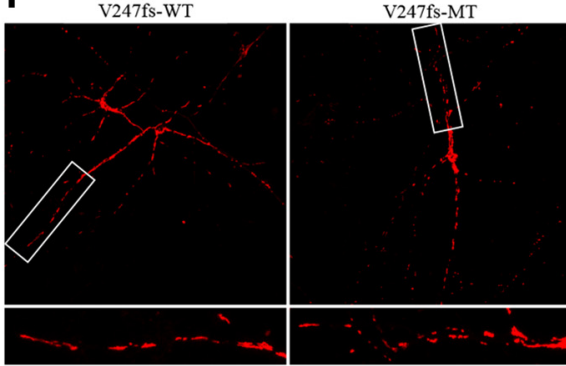

K

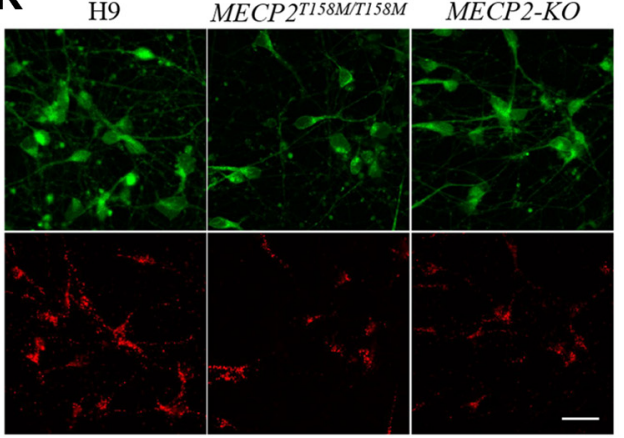

L

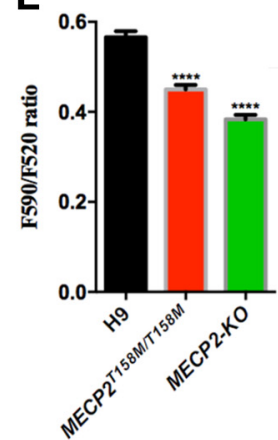

C

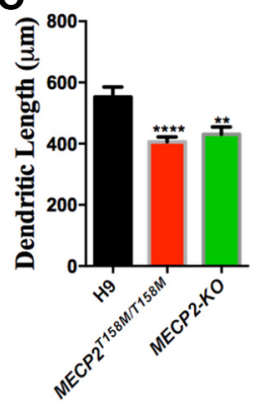

$\mathbf{F}$

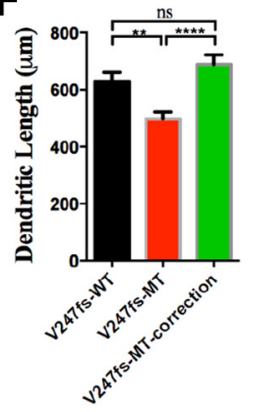

$\mathbf{H}_{3}$

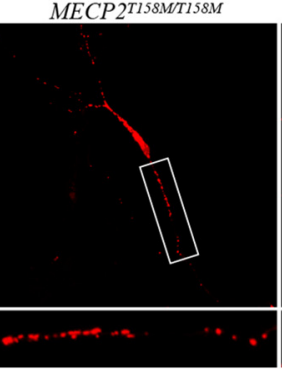

V247fs-MT-Correction

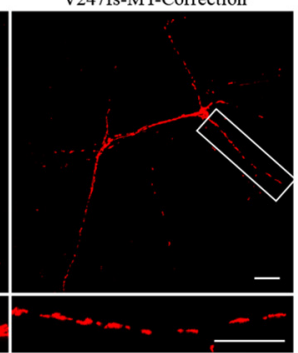

$\mathbf{J}$

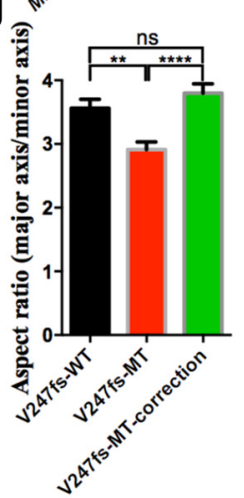

Figure 3. Reduced neuronal growth and impaired mitochondrial health in forebrain neurons differentiated from $M E C P 2 \mathrm{mu}$ tant hESC and iPSC lines. $A$, Representative images of neuronal morphology in Synapsin-GFP-labeled H9, MECP2 ${ }^{T 158 M / T 158 M}$, and MECP2-KOneurons. Scale bar, $20 \mu \mathrm{m}$. B, Sholl analysis of dendritic complexity in Synapsin-GFP-labeled H9, MECP2 ${ }^{T 158 M / T 158 M}$ ' and clone with a 517 bp deletion in the MECP2 coding sequence (MECP2-KO; Fig. $2 A-C$ )

We have previously generated and studied a congenic pair of WT and mutant iPSC lines from RTT patient carrying the V247fs mutation in the MECP2 gene (V247fs-WT and V247fs-MT) (Ananiev et al., 2011; Williams et al., 2014). Although the congenic control is better than age- and gender-matched control, there is still considerable genetic difference between congenic lines due to the difference existed across the

$\leftarrow$

MECP2-KO neurons. Reduced number of neurite intersection indicates reduced complexity. Data points are represented as mean \pm SEM. A total of $47-55$ neurons per line from three independent differentiation experiments were included in the analysis. Two-way ANOVA analysis was followed by Dunnett's post hoc test. ${ }^{* * * *} p<0.0001$, H9 versus MECP2 ${ }^{\text {T158M }}$ T158M. . ${ }^{* * *} p<$ 0.001 , H9 versus MECP2 $2^{\text {T158MT158M }}{ }^{*} p<0.05$, H9 versus

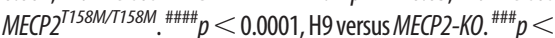
0.001 , H9 versus MECP2-KO. ${ }^{\# \#} p<0.01$, H9 versus MECP2-KO. ${ }^{\#} p<0.05$, H9 versus MECP2-KO. C, Quantification of total neurite length in neurons analyzed in $\boldsymbol{B}$. ${ }^{* * *} p<0.0001$. ${ }^{* *} p<0.01$. D, Representative images of neuronal morphology in Synapsin-GFPlabeled V247fs-WT, V247fs-MT, and V247fs-MT-correction neurons. Scale bar, $20 \mu \mathrm{m}$. $\boldsymbol{E}$, Sholl analysis of dendritic complexity in Synapsin-GFP-labeled V247fs-WT, V247fs-MT, and V247fs-MTcorrection neurons had restored the number of neurite intersections compared with V247fs-MT neurons. Reduced number of neurite intersections indicates reduced complexity. Data points are represented as mean \pm SEM. A total of $47-54$ neurons per line from three independent differentiation experiments were included in the analysis. Two-way ANOVA analysis was followed by Dunnett's post hoc test. ${ }^{* * *}{ }^{* *}<0.0001$, V247fs-MT versus V247fs-WT. ${ }^{* * *} p<0.001$, V247fs-MT versus V247fs-WT. ${ }^{*} p<$

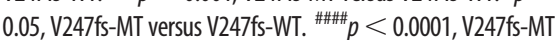
versus V247fs-MT-correction. ${ }^{\# \#} p<0.001$, V247fs-MT versus V247fs-MT-correction. ${ }^{\# \#} p<0.01$, V247fs-MT versus V247fs-MTcorrection. ${ }^{*} p<0.05$, V247fs-MT versus V247fs-MT-correction. $\boldsymbol{F}$, Quantification of total neurite length in neurons analyzed in $\boldsymbol{E}$. ${ }^{* * * *} p<0.0001$. ${ }^{* *} p<0.01$. G, Representative images of H9, MECP2 ${ }^{\text {T158M/T158M }}$, and MECP2-KO neurons expressing SynapsinmitoDsRed2. Enlarged view of the boxed area is shown at the bottom of each image. Scale bars, $20 \mu \mathrm{m}$. $\boldsymbol{H}$, Quantification of mitochondrial aspect ratio in Synapsin-mitoDsRed2-labeled H9, $M E C P 2^{\text {T158MT/158M }}$, and MECP2-KO neurons. A total of $56-60$ neurons per line from three independent differentiation experiments were included in the analysis. ${ }^{* * * *} p<0.0001$. ${ }^{* * *} p<0.001$.I, Representative images of V247fs-WT, V247fs-MT, and V247fsMT-correction neurons expressing Synapsin-mitoDsRed2. Enlarged view of the boxed area is shown at the bottom of each image. Scale bars, $20 \mu \mathrm{m}$. J, Quantification of mitochondrial aspect ratio in Synapsin-mitoDsRed2-labeled V247fs-WT, V247fsMT, and V247fs-MT-correction neurons. A total of 25-33 neurons per line from three independent differentiation experiments were included in the analysis. ${ }^{* * * *} p<0.0001 .{ }^{* *} p<0.01 . \boldsymbol{K}$, Representative images of $J C-10$ dye-stained H9, MECP $2^{1758 M / 158 M}$, and MECP2-KO neurons. Scale bar, $20 \mu \mathrm{m}$. L, Quantification of the ratio of red (F590) and green ( $F 520)$ fluorescence in $\mathrm{H}$, MECP2 ${ }^{\text {T158M }}{ }^{158 M}$, and MECP2-KO neurons. A total of 128-192 neurons per line from three independent differentiation experiments were included in the analysis ${ }^{* * * *} p<0.0001$. Bar graphs, Data are mean \pm SEM. 
A

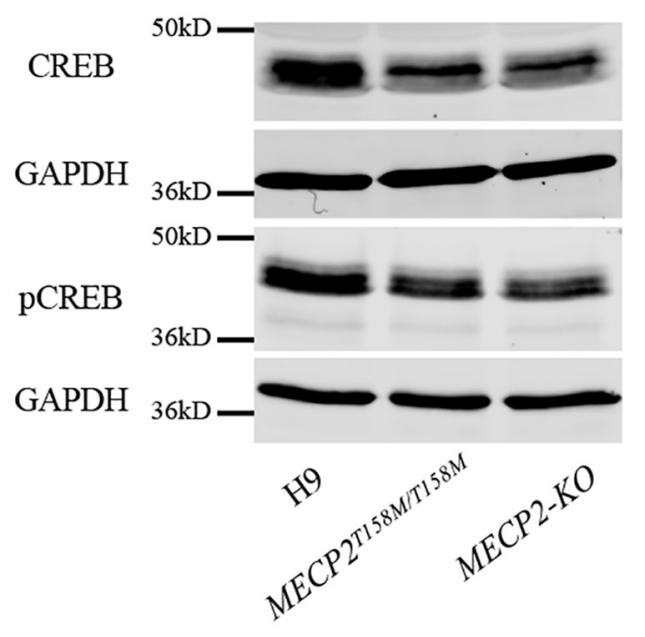

D

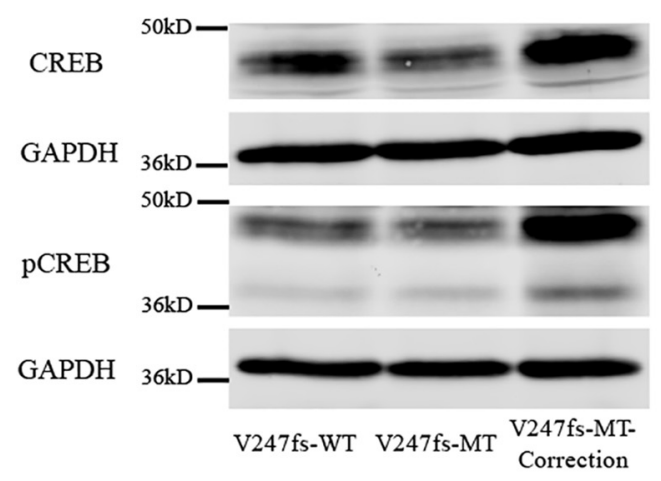

B

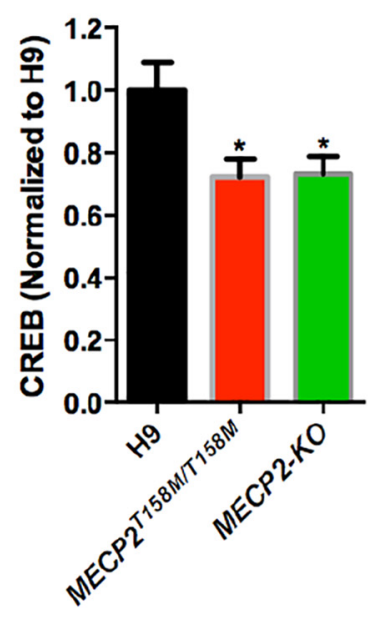

E

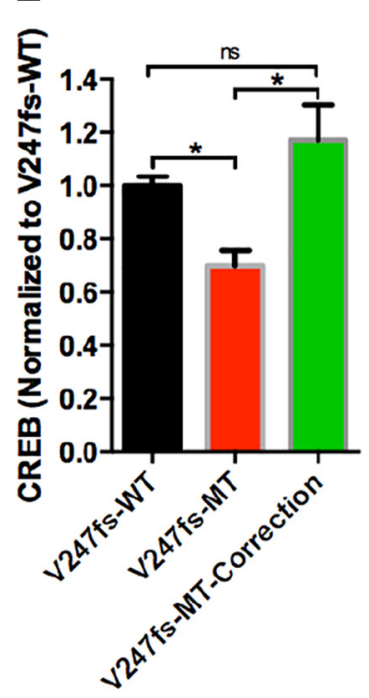

C
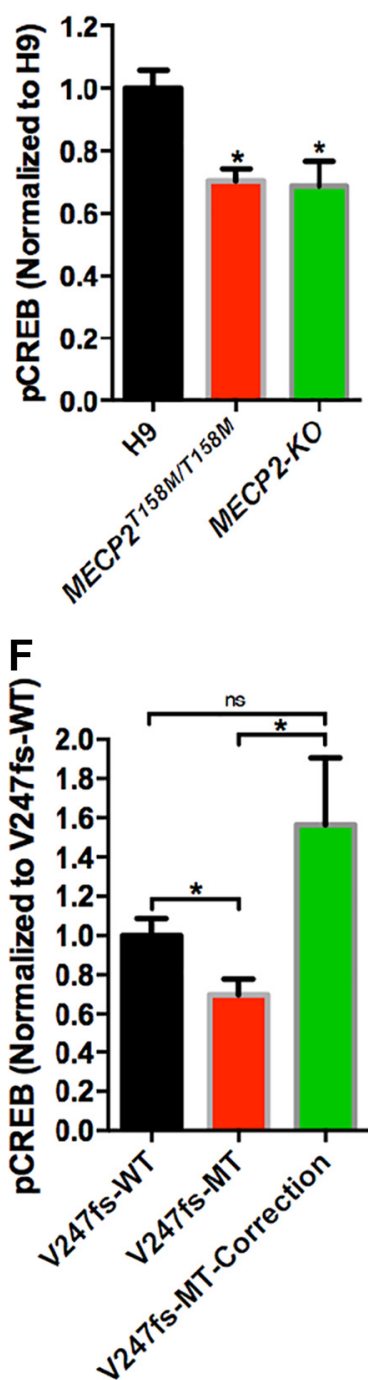

Figure 4. Reduced levels of CREB and pCREB in forebrain neurons differentiated from MECP2 mutant of hESC and iPSC lines. $\boldsymbol{A}-\boldsymbol{C}$, Representative Western blot images ( $\boldsymbol{A}$ ) and quantification of the levels of CREB $(\boldsymbol{B})$ and $\mathrm{pCREB}(\boldsymbol{C})$ in $H 9, M E C P 2^{T 158 M / T 158 M}$, and MECP2-KO neurons. $n=4$ independent differentiation experiments. ${ }^{*} p<0.05$. $\boldsymbol{D}-\boldsymbol{F}$, Representative Western blot images $(\boldsymbol{D})$ and quantification of the levels of CREB $(\boldsymbol{E})$ and pCREB $(\boldsymbol{F})$ in V247fs-WT, V247fs-MT, and V247fs-MT-correction neurons compared with V247fs-MT neurons. $n=3$ independent differentiation experiments. ${ }^{*} p<0.05$. Bar graphs, Data are mean \pm SEM.

entire X chromosome (our congenic lines are clonal for one of the two X chromosomes). To obtain a true isogenic control where the only genetic difference is the MECP2 point mutation, we corrected the V247fs mutation in the V247fs-MT iPSC line using the Cas9, sgRNA77, and donor template containing the WT MECP2 sequence (Fig. $1 F, G$ ). For each genetically targeted line, only karyotypically normal clones were used for subsequent studies (Fig. 2D).

Following a widely used neural differentiation protocol (Chambers et al., 2009), we generated forebrain neurons from three hESC lines (H9, MECP2 $2^{T 158 M / T 158 M}$, and MECP2-KO) and three iPSC lines (V247fs-WT, V247fs-MT, and V247fsMT-correction) (Fig. 1C,H). No obvious difference in the efficiency of neural differentiation was observed between those lines (90\%-95\%; Fig. 1D,I). As expected, no MECP2 expression was detected in either NPs or forebrain neurons differentiated from the MECP2-KO line and the V247fs-MT line (Fig. $1 C, E, H, J)$. Because a previous study showed decreased $\mathrm{MeCP} 2$ protein stability in the Mecp $2^{T 158 A / y}$ mice (Goffin et al., 2011), we examined whether the T158M mutation altered MECP2 protein level in hESC-derived neurons. Indeed, quantitative Western blot analysis revealed a significant reduction in the total MECP2 protein level in MECP2 $2^{\text {T158M/T158M }}$ neurons $(54 \pm 7 \%$ of the level in neurons differentiated from the hESC H9 line, $n=3, p=0.02)$.

Reduced neurite outgrowth, reduced dendritic complexity, and impaired mitochondrial health in forebrain neurons differentiated from MECP2 mutant hESC and iPSC lines To demonstrate the validity of our human stem cell models of RTT, we first looked for hallmark RTT pathologies, such as reduced neuronal growth and dendritic complexity (Armstrong et al., 1995; Kishi and Macklis, 2004; Belichenko et al., 2008, 2009) in forebrain neurons differentiated from MECP2 mutant hESC and iPSC lines. To better visualize and quantify neuronal morphology, we transfected the hESC and iPSC differentiated neurons with a Synapsin-GFP plasmid, which expresses GFP under the control of the synapsin promoter (Fig. $3 A, D$ ). Sholl analysis 
A
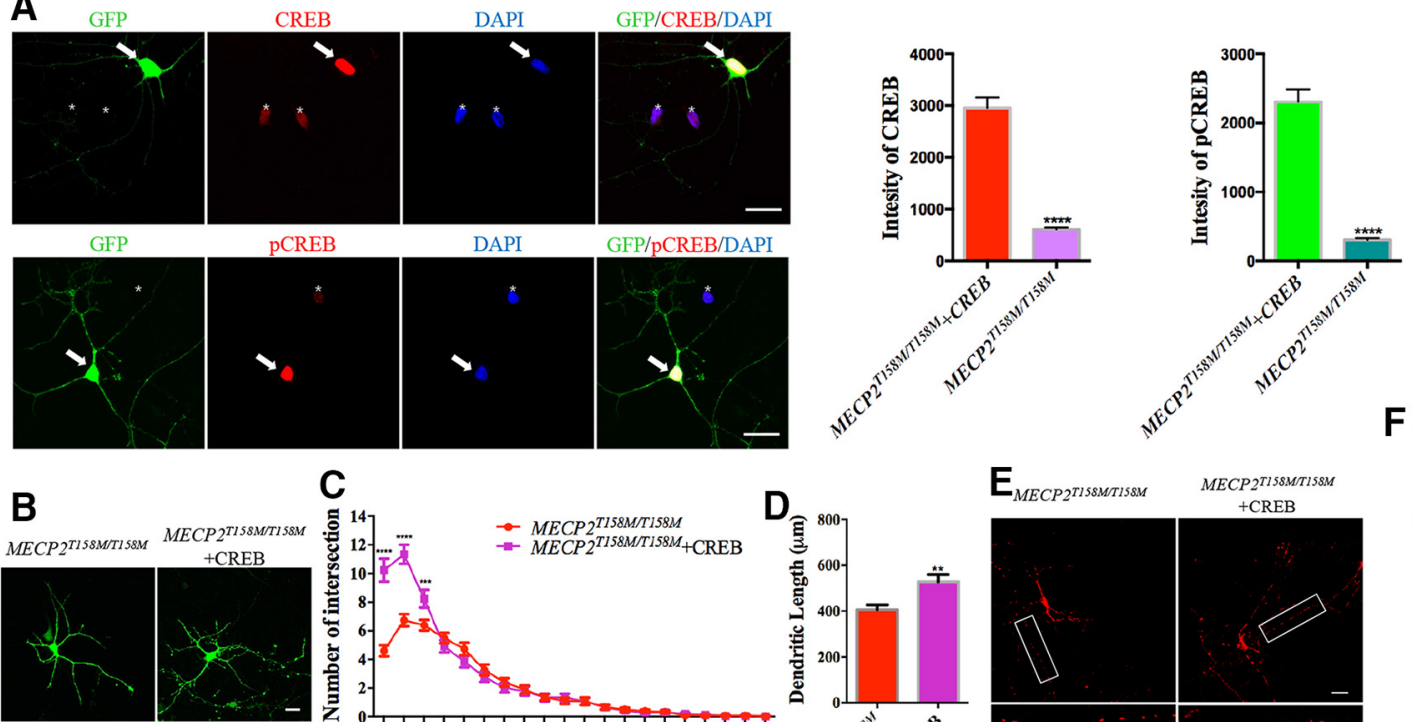

C
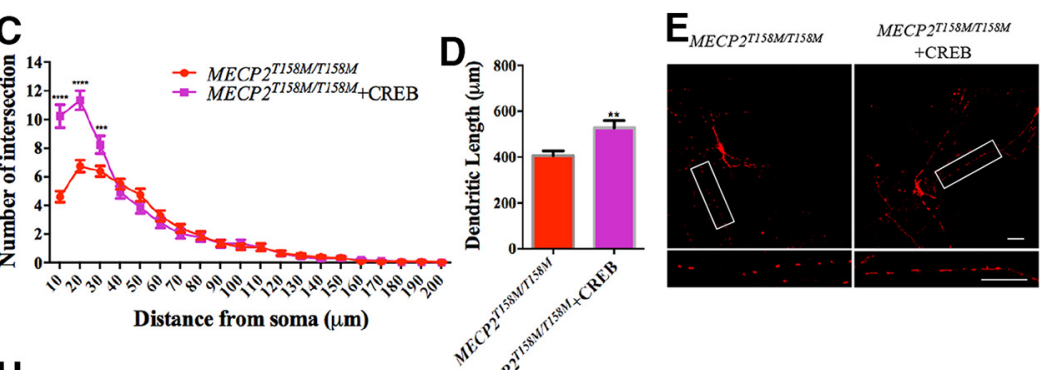

Distance from soma $(\mu \mathrm{m})$

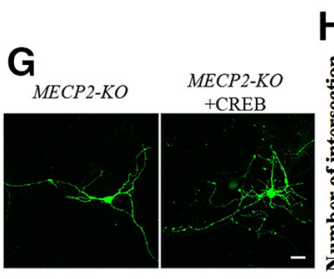

H
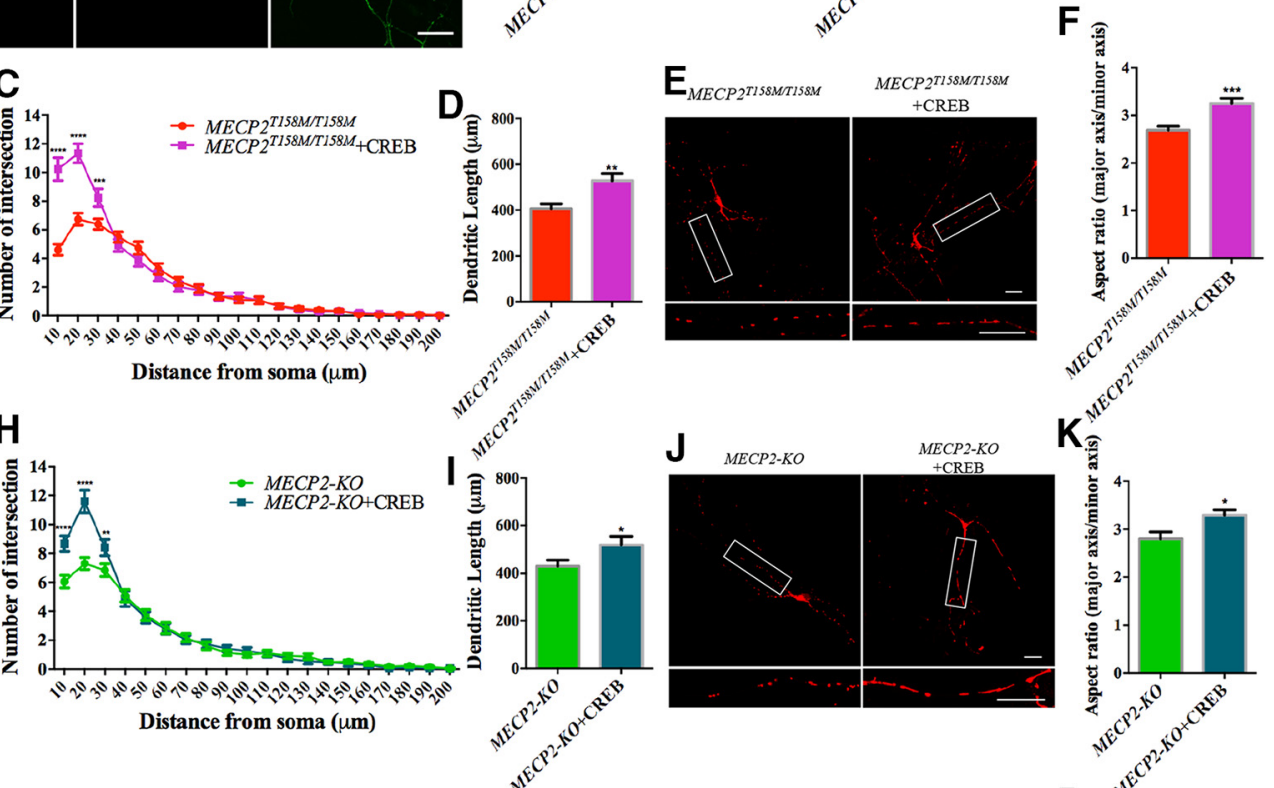

Distance from soma ( $u$ m)

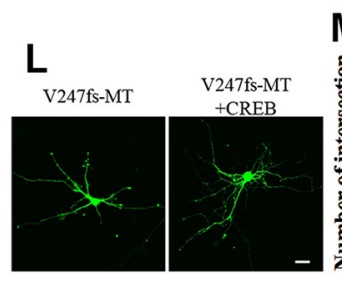

M

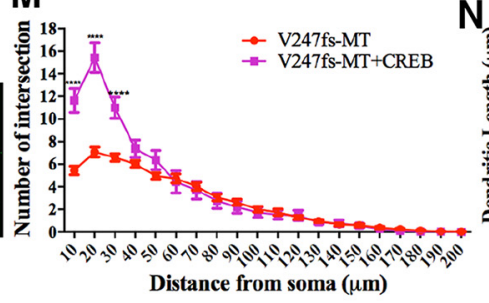

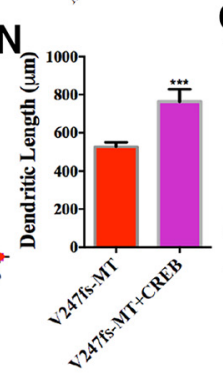

O

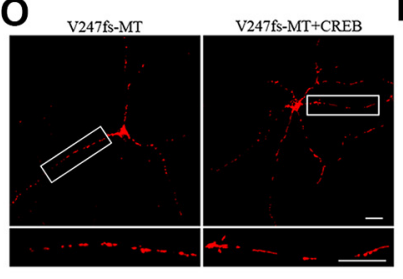

$\mathbf{K}^{\mathrm{s}}$

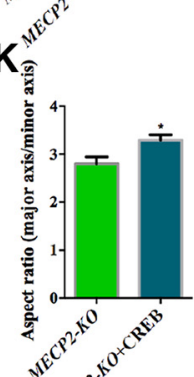

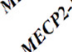

P

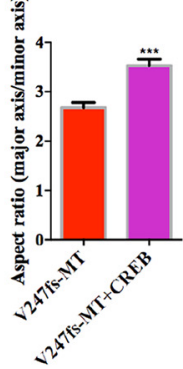

Figure 5. Overexpression of CREB rescues the phenotypes of reduced neuronal growth and mitochondrial fragmentation in MECP2 mutant neurons. $A$, Top, Representative images of SynapsinCREB-Synapsin-GFP (green) labeled MECP2 ${ }^{T 158 M / T 158 M}$ neurons counterstained with DAPI (blue) and immunostained with antibodies specific against CREB (red), and quantification (middle) of the intensity of (REB signal in GFP-positive neurons (one example marked by arrow) and GFP-negative neurons (two examples marked by asterisk). Bottom, Representative images of Synapsin-CREBSynapsin-GFP (green) labeled MECP2 $2^{T 158 M / T 158 M}$ neurons counterstained with DAPI (blue) and immunostained with antibodies specific against pCREB (red), and quantification (right) of the intensity of pCREB signal in GFP-positive neurons (one example marked by arrow) and GFP-negative neurons (one example marked by asterisk). Scale bar, $20 \mu \mathrm{m}$. $\boldsymbol{B}$, Representative images of neuronal morphology in Synapsin-GFP (left) and Synapsin-CREB-Synapsin-GFP (right) expressing MECP2 ${ }^{T 158 M / 7158 M}$ neurons. Scale bar, $20 \mu \mathrm{m}$. C, Sholl analysis of dendritic complexity in Synapsin-GFP or Synapsin-CREB-Synapsin-GFP-expressing MECP2 ${ }^{T 158 M / T 158 M}$ neurons. D, Quantification of total neurite length in Synapsin-GFP or Synapsin-CREB-Synapsin-GFP-expressing MECP2 ${ }^{T 158 M / T 158 M}$ neurons. $C, D$, Data from three independent differentiation experiments were included in analyses. ${ }^{* * *} p<0.0001 .{ }^{* * *} p<0.001 .{ }^{* *} p<0.01$. E, Representative images of Synapsin-mitoDsRed2 (left) and Synapsin-CREB-Synapsin-mitoDsRed2 (right) expressing MECP2 ${ }^{T 158 M / 7158 M}$ neurons. Enlarged view of the boxed area is shown at the bottom of each image. Scale bars, $20 \mu \mathrm{m} . \boldsymbol{F}$, Quantification of mitochondrial aspect ratio in Synapsin-mitoDsRed2 (left) or Synapsin-CREB-Synapsin-mitoDsRed2-expressing MECP2 ${ }^{\text {T158M/T158M }}$ neurons. $n=4$ independent differentiation experiments. ${ }^{* * *} p<0.001 . \mathbf{G}$, Representative images of neuronal morphology in Synapsin-GFP (left) and Synapsin-CREB-Synapsin-GFP (right) expressing MECP2-KO neurons. Scale bar, $20 \mu \mathrm{m}$. $\boldsymbol{H}$, Sholl analysis of dendritic complexity in Synapsin-GFP or Synapsin-CREB-Synapsin-GFP-expressing MECP2-KO neurons. I, Quantification of total neurite length in Synapsin-GFP or Synapsin-CREBSynapsin-GFP-expressing MECP2-KO neurons. $\boldsymbol{H}, \boldsymbol{I}$, Data from three independent differentiation experiments were included in analyses. ${ }^{* * * *} p<0.0001 .{ }^{* *} p<0.01$. ${ }^{*} p<0.05$.J, Representative images of Synapsin-mitoDsRed2 (left) and Synapsin-CREB-Synapsin-mitoDsRed2 (right) expressing MECP2-KO neurons. Enlarged view of the boxed area is shown at the bottom of each image. Scale bars, $20 \mu \mathrm{m}$. $K$, Quantification of mitochondrial aspect ratio in Synapsin-mitoDsRed2 (left) or Synapsin-CREB-Synapsin-mitoDsRed2-expressing MECP2-KO neurons. $n=4$ independent differentiation experiments. ${ }^{* * *} p<0.001$. Scale bar, $20 \mu \mathrm{m}$. L, Representative images of neuronal morphology in Synapsin-GFP (left) and Synapsin-CREB-Synapsin-GFP (right) expressing V247fs-MT neurons. Scale bar, $20 \mu \mathrm{m} . \boldsymbol{M}$, Sholl analysis of dendritic complexity in Synapsin-GFP or Synapsin-CREB-Synapsin-GFP-expressing V247fs-MT neurons. N, Quantification of total neurite length in Synapsin-GFP or Synapsin-CREB-Synapsin-GFP-expressing V247fs-MT neurons. $M, N$, Data from three independent differentiation experiments were included in analyses. ${ }^{* * * *} p<0.0001$. ${ }^{* * *} p<$ 0.001. $\mathbf{O}$, Representative images of Synapsin-mitoDsRed2 (left) and Synapsin-CREB-Synapsin-mitoDsRed2 (right) expressing V247fs-MT neurons. Enlarged view of the boxed area is shown at the bottom of each image. Scale bars, $20 \mu \mathrm{m}$. P, Quantification of mitochondrial aspect ratio in Synapsin-mitoDsRed2 (left) or Synapsin-CREB-Synapsin-mitoDsRed2-expressing V247fs-MT neurons. $n=3$ independent differentiation experiments. ${ }^{* * *} p<0.001$. Bar graphs, Data are mean \pm SEM. 


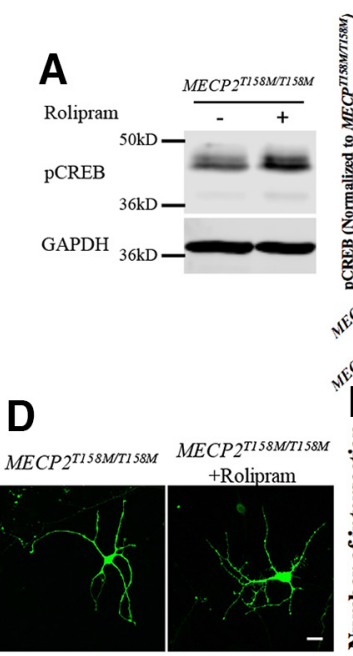

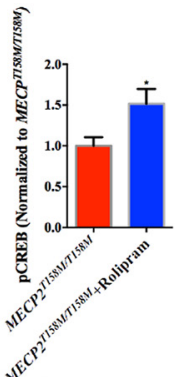

E
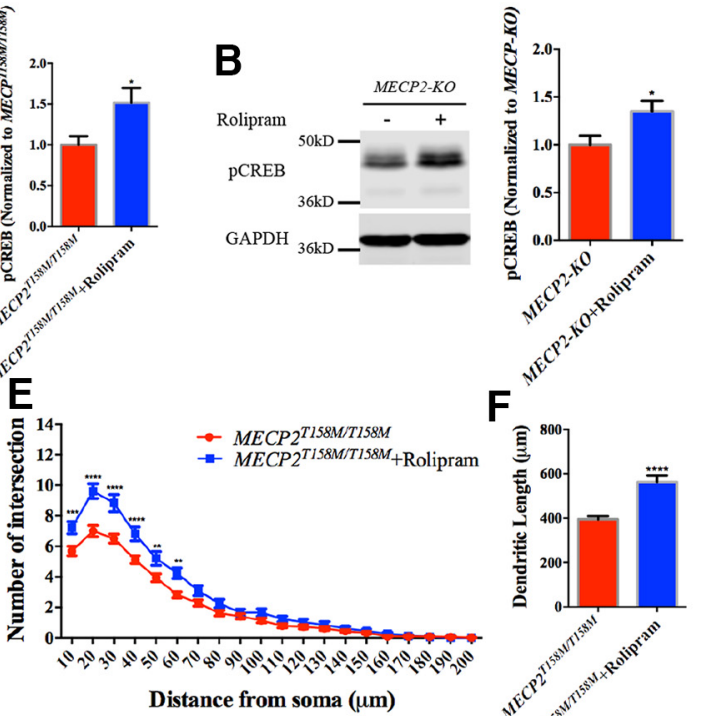

$F^{3}$

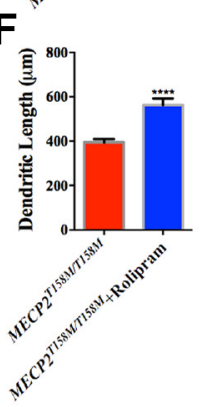

G
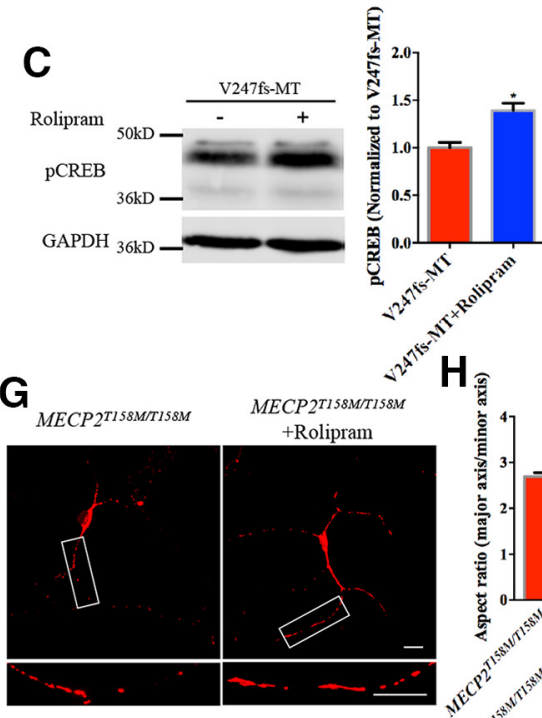

$\mathrm{H}_{\overparen{g}}$

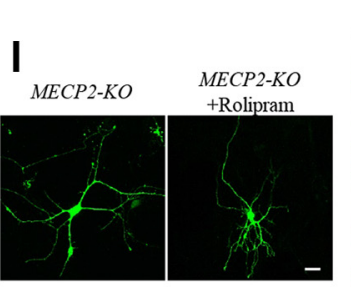

J

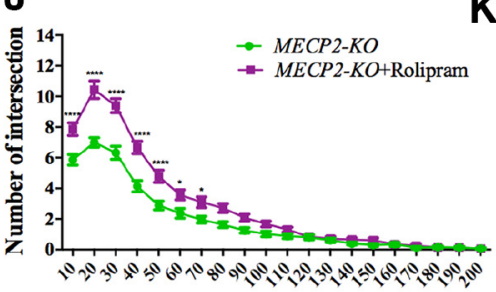

Distance from soma $(\mu \mathrm{m})$

O
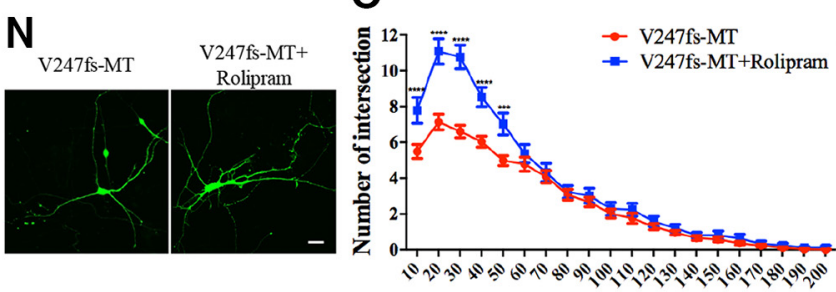

Distance from soma $(\mu \mathrm{m})$

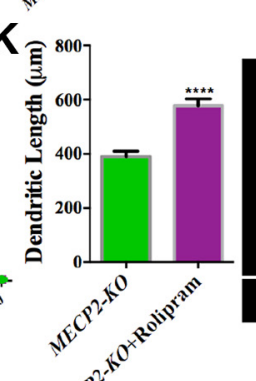

$\mathbf{L}_{M E C P 2-K O}$
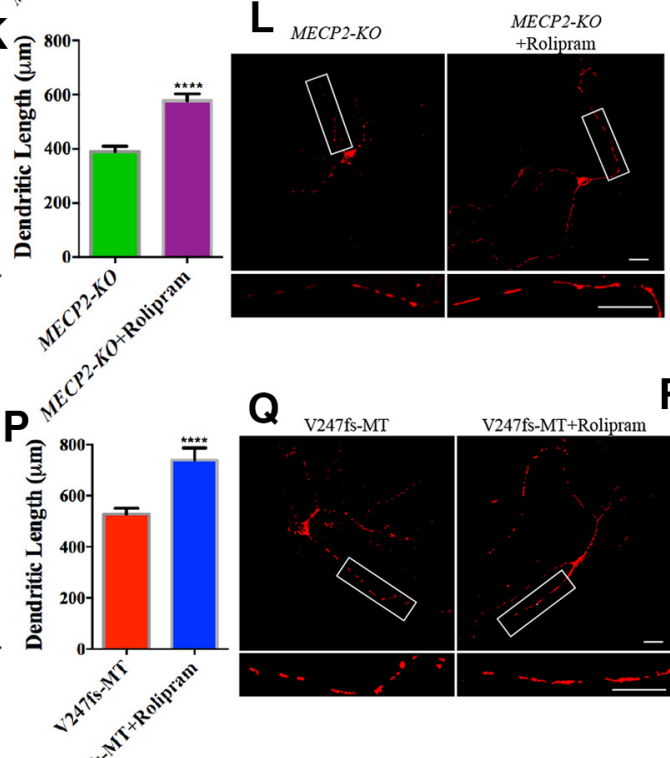

Q

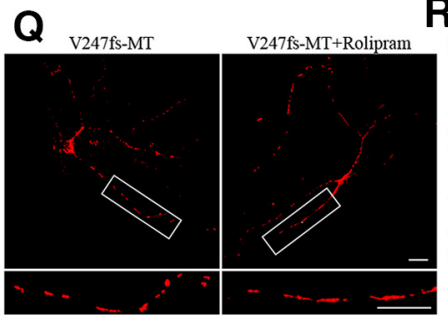

$\mathbf{R}$

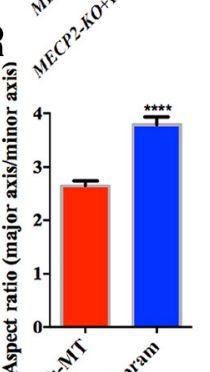

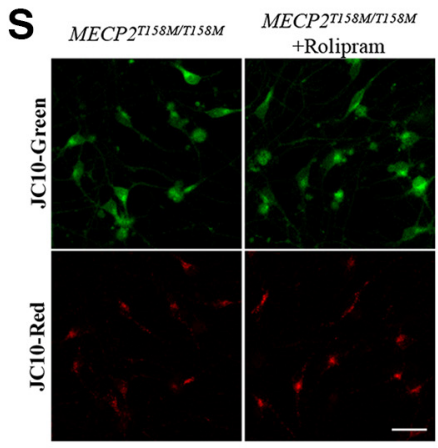
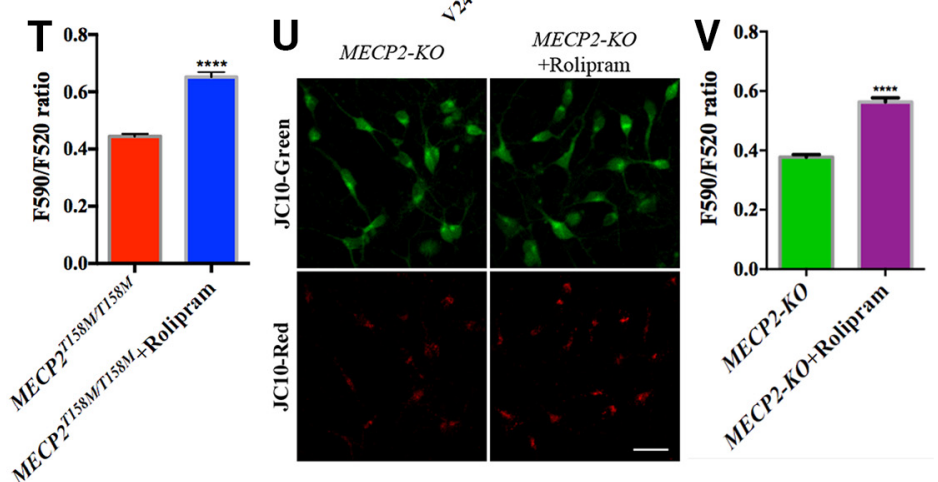

Figure 6. Rolipram activates CREB signaling and rescues the phenotypes of reduced neuronal growth, mitochondrial fragmentation, and reduced MMP in $M E C P 2$ mutant neurons. $A$, Representative Western blot images (left) and quantification (right) of $p C R E B$ level in vehicle- and rolipram-treated MECP2 ${ }^{T 158 M / T 158 M}$ neurons. $n=4$ independent differentiation experiments. ${ }^{*} p<0.05$. $B$, Representative Western blot images (left) and quantification (right) of pCREB level in vehicle- and rolipram-treated MECP2-KO neurons. $n=4$ independent differentiation experiments. ${ }^{*} p<0.05$. C, Representative Western blot images (left) and quantification (right) of $p$ CREB level in vehicle- and rolipram-treated V247fs-MT neurons. $n=3$ independent differentiation experiments. ${ }^{*} P<0.05$. D, Representative images of Synapsin-GFP-labeled MECP2 ${ }^{T 158 M / T 158 M}$ neurons treated with vehicle (left) or rolipram (right). Scale bar, $20 \mu \mathrm{m}$. $\boldsymbol{E}$, $\boldsymbol{F}$, Sholl analysis of dendritic complexity $(\boldsymbol{E})$ and quantification of total neurite length $(\boldsymbol{F})$ in Synapsin-GFP-labeled MECP2 ${ }^{T 158 M / T 158 M}$ neurons treated with vehicle or rolipram. $n=3$ independent differentiation experiments. ${ }^{* * *} p<0.0001 .{ }^{* * *} p<0.001$. ${ }^{* *} p<0.01$. G, Representative images of Synapsin-mitoDsRed2-expressing MECP2 ${ }^{T 158 M / T 158 M}$ neurons treated with vehicle (left) or rolipram (right). Scale bar, 20 $\mu \mathrm{m} . \boldsymbol{H}$, Quantification of mitochondrial aspect ratio in Synapsin-mitoDsRed2-expressing MECP2 ${ }^{T 158 M / 7158 M}$ neurons treated with vehicle or rolipram. $n=3$ independent differentiation experiments. ${ }^{* * *} p<0.0001$. I, Representative images of Synapsin-GFP-labeled MECP2-KO neurons treated with vehicle (left) or rolipram (right). Scale bar, $20 \mu \mathrm{m}$. (Figure legend continues.) 
on GFP-positive neurons of each genotype revealed significantly reduced dendritic arborization and total neurite length in $M E C P 2^{T 158 M / T 158 M}$ and MECP2-KO neurons compared with $\mathrm{H} 9$ neurons (Fig. 3 B, C), and in V247fs-MT neurons compared with both the congenic control (V247fs-WT) and the isogenic control (V247fs-MT-correction) (Fig. 3E,F). In addition to neuronal morphology, we examined another well-documented, yet less studied, cellular pathology in RTT cells, mitochondrial defect (Eeg-Olofsson et al., 1990; Coker and Melnyk, 1991; Dotti et al., 1993; Cornford et al., 1994; Jugloff et al., 2005; Grosser et al., 2012; Gold et al., 2014). To better visualize and quantify mitochondrial morphology, we transfected the hESC and iPSC differentiated neurons with a Synapsin-mitoDsRed2 plasmid, which expresses a version of DsRed2 localized specifically to the mitochondrion (Fig. 3G,I). Detailed quantification of the shape of mitoDsRed2 puncta (Fig. 3G,I, enlarged inset) revealed significantly reduced aspect ratio in $M E C P 2^{T 158 M / T 158 M}$ and MECP2-KO neurons compared with $\mathrm{H} 9$ neurons (Fig. $3 \mathrm{H}$ ), and in V247fs-MT neurons compared with both the congenic control (V247fs-WT) and the isogenic control (V247fs-MT-correction) (Fig. $3 J$ ), suggesting that the mitochondria were more fragmented in MECP2 mutant neurons. To establish more quantitative measurement of mitochondrial deficit in RTT cells, we used the JC-10 assay (Abcam) to examine the mitochondrial membrane potential (MMP) in hESC differentiated forebrain neurons (Fig. $3 \mathrm{~K}$ ). The JC-10 dye is capable of selectively entering into mitochondria and reversibly changing its color from green to red as membrane potential increases. This property is due to the reversible formation of JC-10 aggregates upon membrane polarization that causes shifts in emitted light from the wavelength of $520 \mathrm{~nm}$ (emission of JC-10 monomeric form) to the wavelength of $590 \mathrm{~nm}$ (emission of JC-10 aggregate form). Therefore, the ratio of fluorescence emission at $590 \mathrm{~nm}$ over fluorescence emission at $520 \mathrm{~nm}$ (F590/F520) directly correlates with the MMP. Our analysis found a significantly decreased ratio of red/green fluorescence in the MECP2 $2^{T 158 M / T 158 M}$ and MECP2-KO neurons compared with the WT neurons (Fig. 3L), indicating a less polarized MMP (a sign of less healthy mitochondria) in MECP2 mutant neurons. Together, increased fragmentation and reduced

\section{$\leftarrow$}

(Figure legend continued.) $\boldsymbol{J}, \boldsymbol{K}$, Sholl analysis of dendritic complexity $(\boldsymbol{J})$ and quantification of total neurite length $(\boldsymbol{K})$ in Synapsin-GFP-labeled MECP2-KO neurons treated with vehicle or rolipram. $n=3$ independent differentiation experiments. ${ }^{* * *} p<0.0001 .{ }^{*} p<0.05$. $L$, Representative images of Synapsin-mitoDsRed2-expressing MECP2-KO neurons treated with vehicle (left) or rolipram (right). Scale bar, $20 \mu \mathrm{m}$. M, Quantification of mitochondrial aspect ratio in Synapsin-mitoDsRed2-expressing MECP2-KO neurons treated with vehicle or rolipram. $n=3$ independent differentiation experiments. ${ }^{* *} p<0.01$. $N$, Representative images of Synapsin-GFP-labeled V247fs-MT neurons treated with vehicle (left) or rolipram (right). Scale bar, $20 \mu \mathrm{m} . \mathbf{0}, \boldsymbol{P}$, Sholl analysis of dendritic complexity ( $\mathbf{0}$ ) and quantification of total neurite length $(\boldsymbol{P})$ in Synapsin-GFP-labeled V247fs-MT neurons treated with vehicle or rolipram. $n=3$ independent differentiation experiments. ${ }^{* * *} p<0.0001 .{ }^{* * *} p<0.001$. $\boldsymbol{Q}$, Representative images of Synapsin-mitoDsRed2-expressing V247fs-MT neurons treated with vehicle (left) or rolipram (right). Scale bar, $20 \mu \mathrm{m}$. $\boldsymbol{R}$, Quantification of mitochondrial aspect ratio in SynapsinmitoDsRed2-expressing V247fs-MT neurons treated with vehicle or rolipram. $n=3$ independent differentiation experiments. ${ }^{* * * *} p<0.0001$. S, Representative images of JC-10 dyestained MECP2 $2^{T 158 M / T 158 M}$ neurons treated with vehicle (left) or rolipram (right). Scale bar, $20 \mu \mathrm{m}$. $T$, Quantification of the ratio of red (F590) and green (F520) fluorescence in $M E C P 2^{T 158 M / T 158 M}$ neurons treated with vehicle or rolipram. $n=3$ independent differentiation experiments. ${ }^{* * *} p<0.0001$. $U$, Representative images of JC-10 dye-stained MECP2-KO neurons treated with vehicle (left) or rolipram (right). Scale bar, $20 \mu \mathrm{m}$. V, Quantification of the ratio of red (F590) and green (F520) fluorescence in MECP2-KO neurons treated with vehicle or rolipram. $n=3$ independent differentiation experiments. ${ }^{* * *} p<0.0001$. Bar graphs, Data are mean \pm SEM.
MMP provide two quantitative measures of compromised mitochondrial health in RTT neurons.

\section{Reduced CREB level underlies defects in neuronal growth and mitochondrial health in MECP2 mutant neurons}

To further elucidate the molecular mechanism underlying the cellular phenotypes of abnormal neuronal morphology and mitochondrial deficit in MECP2 mutant neurons, we focused on $\mathrm{CREB}$, a transcription factor known to indirectly regulate $\mathrm{MeCP} 2$ level (Klein et al., 2007), directly interact with MeCP2 (Chahrour et al., 2008), and control the expression of numerous genes that regulate dendritic growth (Redmond et al., 2002; Wayman et al., 2006) and mitochondrial function (Ryu et al., 2005). Western blot analysis revealed significantly reduced levels of both total CREB and phosphorylated CREB (pCREB, a signature of CREB activation) in MECP2 $2^{T 158 M / T 158 M}$ and $M E C P 2-K O$ neurons compared with $\mathrm{H} 9$ neurons (Fig. 4A-C), and in V247fs-MT neurons compared with both the congenic control (V247fs-WT) and the isogenic control (V247fs-MT-correction) (Fig. 4D-F).

If reduced neuronal growth, reduced dendritic complexity, and abnormal mitochondrial morphology in MECP2 mutant neuron are due to decreased CREB expression, overexpression of CREB should rescue those phenotypes. To that end, we transfected MECP2 mutant neurons with a plasmid expressing CREB and GFP under the control of the synapsin promoter, and used immunostaining with antibodies specific against CREB and pCREB to confirm the significant increase of CREB and pCREB levels in GFP-positive neurons (Fig. 5A). More importantly, CREB overexpression significantly increased dendritic complexity and total neurite length in $M E C P 2^{T 158 M / T 158 M}$ neurons (Fig. $5 B-D$ ), MECP2-KO neurons (Fig. 5G-I), and V247fs-MT neurons (Fig. $5 L-N$ ). To access the effect of CREB overexpression on mitochondrial morphology, we transfected MECP2 mutant neurons with a plasmid expressing both CREB and mitoDsRed2 under the synapsin promoter. Consistent with its effect on neuronal morphology, CREB overexpression also significantly reduced mitochondria fragmentation in $M E C P 2^{T 158 M / T 158 M}$ neurons (Fig. $5 E, F$ ), MECP2-KO neurons (Fig. $5 \mathrm{~J}, K$ ), and V247fs-MT neurons (Fig. 5O,P).

Rolipram, a selective phosphodiesterase 4 (PDE-4) inhibitor, is known to increase the level of cAMP that consequently increases phosphorylation of CREB (Li et al., 2009). Thus, we measured the level of pCREB in MECP2 $2^{T 158 M / T 158 M}, M E C P 2-K O$, and V247fs-MT-correction neurons in the presence of $40 \mu \mathrm{M}$ rolipram for $48 \mathrm{~h}$. Western blot analysis detected significantly increased levels of pCREB in those MECP2 mutant neurons treated with rolipram (Fig. $6 A-C$ ). Moreover, rolipram treatment rescued the phenotypes of reduced dendritic complexity, reduced total neurite length, and fragmented mitochondria in $M E C P 2^{T 158 M / T 158 M}$ neurons (Fig. $6 D-H$ ), MECP2-KO neurons (Fig. 6I-M), and V247fs-MT neurons (Fig. $6 N-R$ ). In addition, rolipram treatment was able to rescue the MMP deficit in $M E C P 2^{T 158 M / T 158 M}$ and MECP2-KO neurons (Fig. $6 S-V$ ). Together, these results strongly suggest that reduced CREB expression is a key contributor to the phenotypes of reduced neuronal growth and impaired mitochondrial health in MECP2 mutant neurons.

Previous studies have identified WNT2 as a CREB target gene involved in regulating dendritic outgrowth (Wayman et al., 2006). Interestingly, we observed a significant decrease in WNT2 mRNA level in MECP2 mutant neurons $(53 \pm 8 \%$ of the WT level in MECP2 $2^{T 158 M / T 158 M}$ neurons, $n=7, p=0.007$; $51 \pm 9 \%$ of the WT level in MECP2-KO neurons, $n=7, p=$ 
A

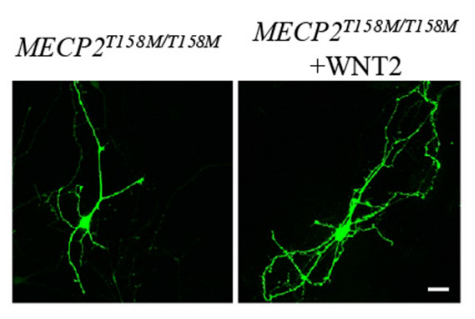

D
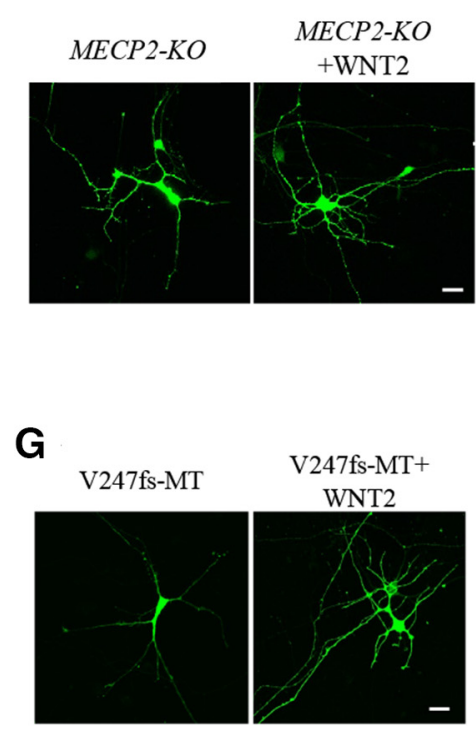

B

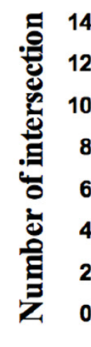

E

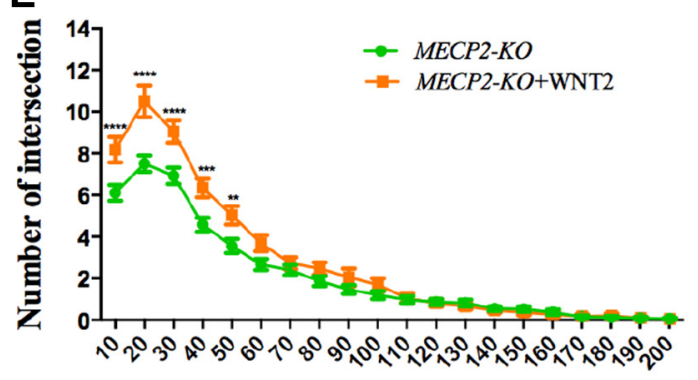

H

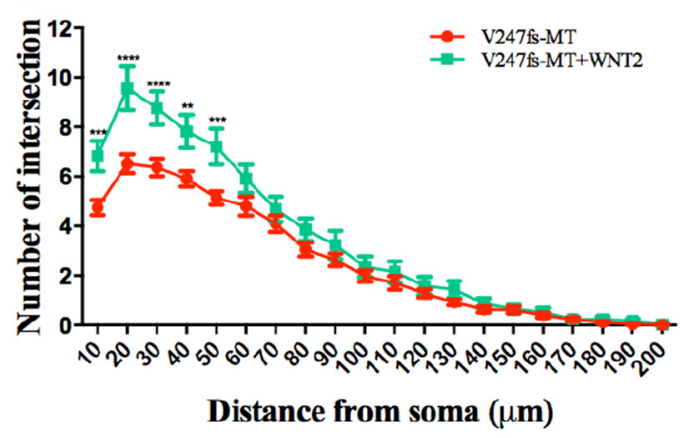

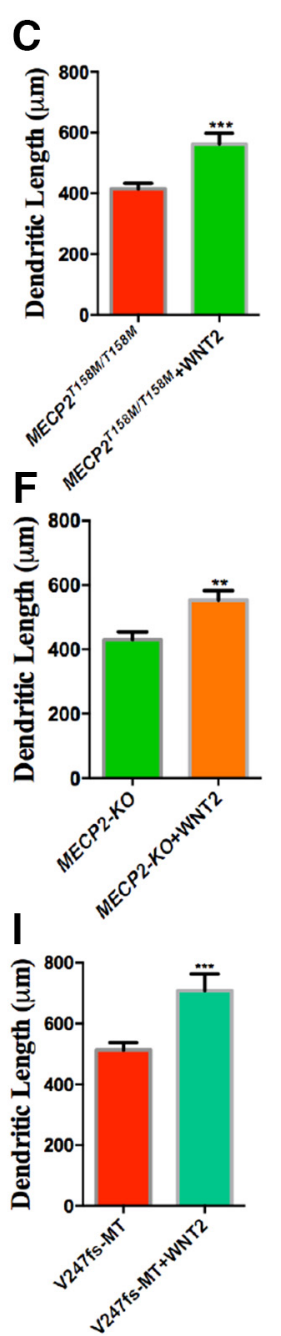

Figure 7. Overexpression of WNT2 rescues the phenotype of reduced neuronal growth in MECP2 mutant neurons. $A$, Representative images of neuronal morphology in Synapsin-GFP (left) and Synapsin-WNT2-Synapsin-GFP (right) expressing MECP2 ${ }^{\text {T158M/T158M }}$ neurons. Scale bar, $20 \mu \mathrm{m}$. B, Sholl analysis of dendritic complexity in Synapsin-GFP or Synapsin-WNT2-Synapsin-GFPexpressing MECP2 ${ }^{T 158 M / T 158 M}$ neurons. C, Quantification of total neurite length in Synapsin-GFP or Synapsin-WNT2-Synapsin-GFP-expressing MECP2 ${ }^{T 158 M / T 158 M}$ neurons. B, C, Data from three independent differentiation experiments were included in analyses. ${ }^{* *} p<0.001$. ${ }^{* *} p<0.01$. ${ }^{*} p<0.05$. D, Representative images of neuronal morphology in Synapsin-GFP (left) and Synapsin-WNT2-Synapsin-GFP (right) expressing MECP2-KO neurons. Scale bar, $20 \mu \mathrm{m}$. E, Sholl analysis of dendritic complexity in Synapsin-GFP or Synapsin-WNT2-Synapsin-GFP-expressing MECP2-KO neurons. $\boldsymbol{F}$, Quantification of total neurite length in Synapsin-GFP or Synapsin-WNT2-Synapsin-GFP-expressing MECP2-KO neurons. $\boldsymbol{E}, \boldsymbol{F}$, Data from three independent differentiation experiments were included in analyses. ${ }^{* * *} p<0.0001 .{ }^{* * *} p<0.001 .{ }^{* *} p<0.01$. G, Representative images of neuronal morphology in Synapsin-GFP (left) and Synapsin-WNT2-Synapsin-GFP (right) expressing V247fs-MT neurons. Scale bar, $20 \mu \mathrm{m}$. H, Sholl analysis of dendritic complexity in Synapsin-GFP or Synapsin-WNT2-Synapsin-GFP-expressing V247fs-MT neurons. I, Quantification of total neurite length in Synapsin-GFP or Synapsin-WNT2-Synapsin-GFP-expressing V247fs-MT neurons. $\boldsymbol{H}$, I, Data from three independent differentiation experiments were included in analyses. ${ }^{* * *} p<0.001 .{ }^{* *} p<0.01$. ${ }^{* *} p<0.01$. Bar graphs, Data are mean \pm SEM.

0.005). Furthermore, MECP2 mutant neurons transfected with Synapsin-WNT2 plasmid (expressing WNT2 under the control of synapsin promoter) exhibited increased dendritic complexity and total neurite length (Fig. 7), suggesting that reduced WNT2 expression is a downstream effector of reduced CREB signaling in mediating neuronal growth MECP2 mutant neurons.

\section{Chronic rolipram treatment alleviates behavioral symptoms} in female Mecp2 heterozygous mice

To further explore whether manipulating CREB signaling may be a valid therapeutic strategy, we evaluated the efficacy of chronic rolipram treatment in female Mecp2 heterozygous mice $\left(M e c p 2^{-/+}\right)$. Compared with the more studied male Mecp2 knock-out mice $\left(M e c p 2^{-/ y}\right)$, the $M e c p 2^{-/+}$mice show delayed disease onset, slower disease progression, and more variation in phenotypic severity from animal to animal (due to random $\mathrm{X}$ chromosome inactivation in individual animals). Yet, the $M e c p 2^{-/+}$mice more accurately reflect the genetics of RTT patients, as the majority of RTT patients are females. Consistent with our findings in human RTT neurons, Western blot analysis revealed significantly reduced levels of both total CREB and phosphorylated CREB in the cortex of female $M e c p 2^{-/+}$mice (Fig. 8A). This result provided further rationale for assessing the therapeutic potential of enhanced CREB signaling in female $M e c p 2^{-/+}$mice. The 4- to 6-month-old Mecp $2^{-/+}$mice were given either vehicle or rolipram $(0.03 \mathrm{mg} / \mathrm{kg})$ daily for a period of 8 weeks. Behavioral tests were performed after the stop of treatment. In the open field test, the vehicle-treated $M e c p 2^{-/+}$mice demonstrated reduced locomotor activity as measured by the 

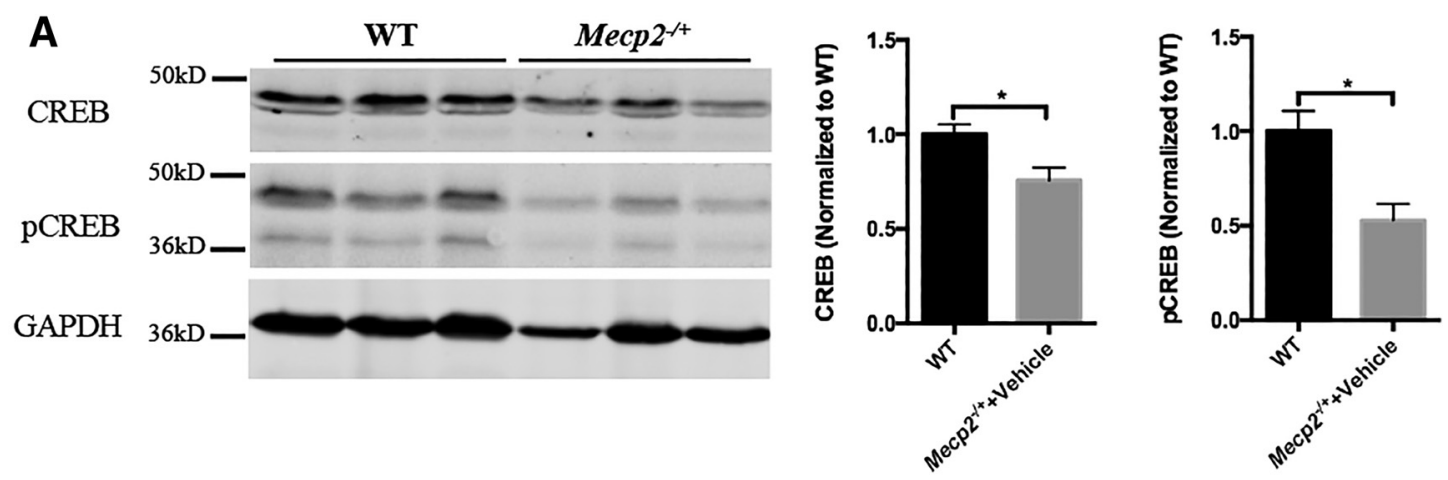

B

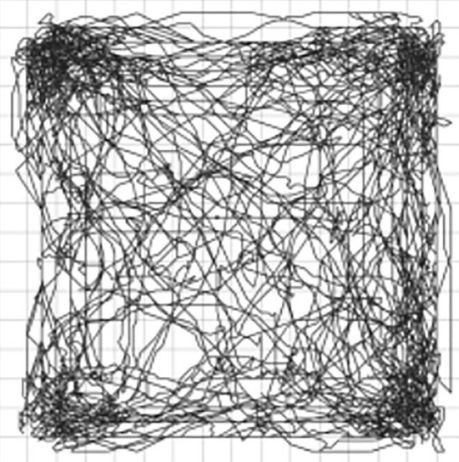

C

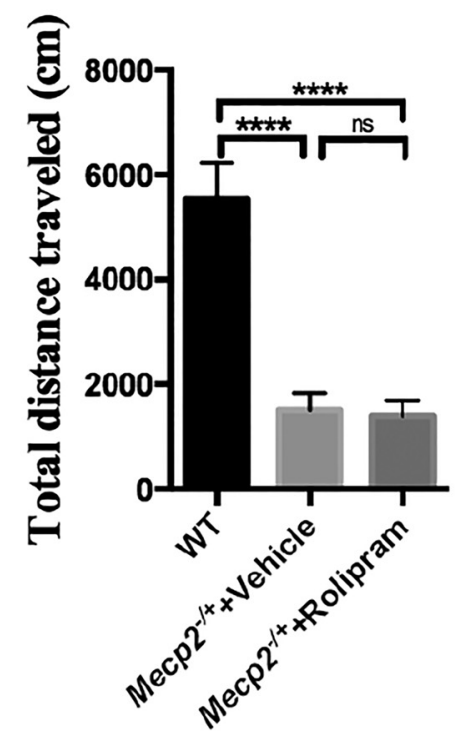

Mecp $2^{-/ 4}+$ Vehicle

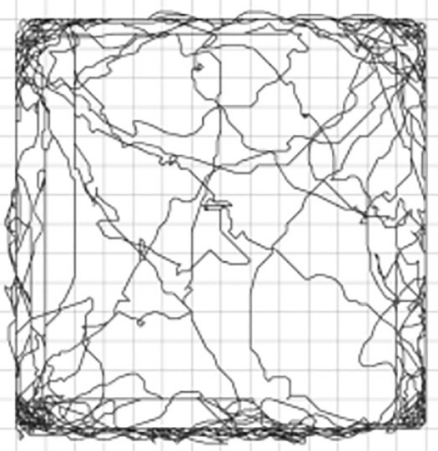

D

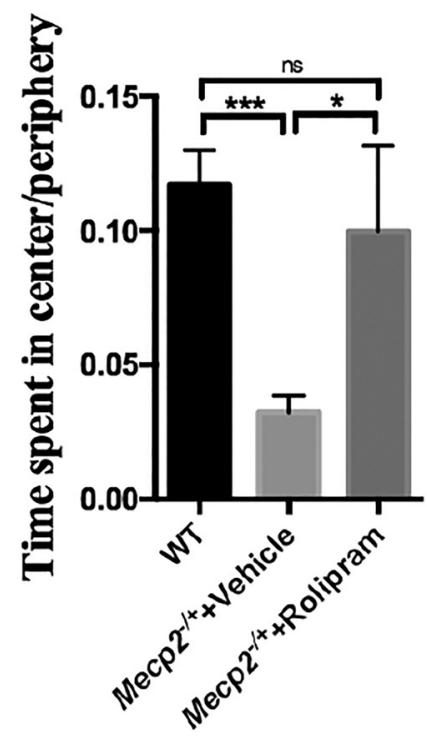

Mecp $^{-/ 4}+$ Rolipram

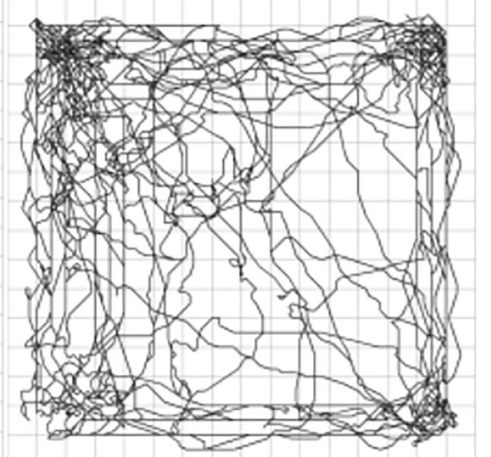

E

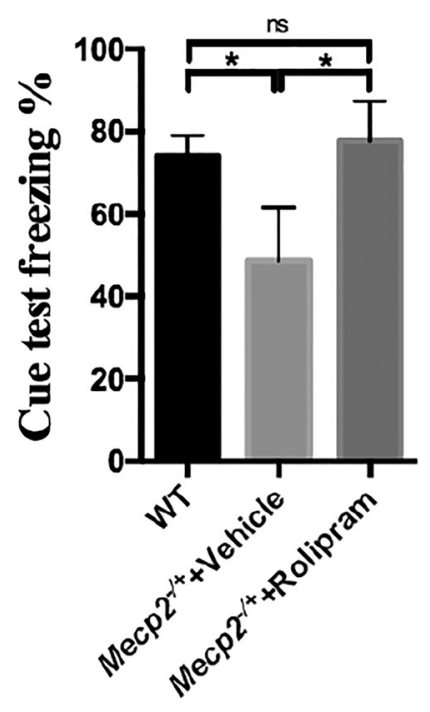

Figure 8. Chronic rolipram treatment alleviates behavioral phenotypes in female $M e c p 2^{-/+}$mice. $A$, Representative Western blot images and quantification of the levels CREB and $p C R E B$ in the cortex of WT and Mecp $2^{-/+}$mice. $B$, Representative movement tracking plots from each group of mice in the open field test. $\boldsymbol{C}$, Quantification of the total distances traveled by each group of mice during the testing period in the open field test. $D$, Quantification of the ratio of time spent in the center over time spent in the periphery of the testing area by each group of mice during the testing period in the open field test. $\boldsymbol{E}$, Quantification of the percentage of the time spent frozen by each group of mice in the cue test of the fear conditioning paradigm. Eight to 11 mice from each group of mice were tested. ${ }^{* * * *} p<0.0001 .{ }^{* *} p<0.001 .{ }^{*} p<0.05$. Bar graphs, Data are mean \pm SEM.

total distance traveled (Fig. $8 B, C$ ), and increased level of anxiety as measured by reduced ratio of time spent in the center of the field (Fig. $8 D$ ). Although it did not improve the locomotor activity in $M e c p 2^{-/+}$mice (Fig. $8 B, C$ ), chronic rolipram treatment reversed the anxiety-related behavior in $M e c p 2^{-/+}$mice (Fig. $8 D)$. Furthermore, chronic rolipram treatment significantly improved the performance of $M e c p 2^{-/+}$mice in the cue test of the fear conditioning paradigm (Fig. $8 E$ ). Results from this series of animal experiments suggest that manipulation of CREB signaling has potential to become a valid therapeutic target in treating RTT.

\section{Discussion}

Genetically engineered hESCs and patient-specific iPSCs are powerful models for studying human diseases, as they offer direct 
human relevance and are readily accessible for molecular and pharmacological manipulations. Yet, studies using hESCs and iPSCs often face two challenges: (1) gene deletion in hESCs often leads to the complete loss of expression of a given disease gene, which could be different from the expression of a missense mutation found in disease; and (2) patient-specific iPSC lines and gender- and age-matched control iPSC lines differ significantly in genetic background, leading to substantial phenotypic variations. Specific for RTT research, although hESC lines lacking MECP2 have been created (Li et al., 2013), no hESC lines carrying disease causing missense MECP2 point mutations have been reported. Taking advantage of nonrandom (clonal) X chromosome inactivation among female iPSC lines, we previously generated congenic pairs of WT and mutant RTT iPSC lines from the same patients (Ananiev et al., 2011; Williams et al., 2014). Yet, the congenic control is not as ideal as the isogenic control because the congenic control transcribes an entirely different X chromosome. To establish a combination of hESC lines and iPSC lines that address these limitations, we used CRISPR/Cas9-mediated gene editing to generate hESC lines either carrying either the common disease causing missense mutation (T158M) in the endogenous $M E C P 2$ gene $\left(M E C P 2^{T 158 M / T 158 M}\right)$ or a random deletion of MECP2 (MECP2-KO), as well as to correct the V247fs mutation in the V247fs-MT iPSC line (V247fs-MT-correction) in the current study. Compared with their respective isogenic controls, the $M E C P 2^{T 158 M / T 158 M}, M E C P 2-K O$, V247fs-MT-correction neurons consistently displayed the hallmark RTT pathology of reduced neuronal growth as measured by the length and complexity of neurites, providing strong evidence that these are valid cellular models of RTT. Moreover, we extended our cellular analysis to mitochondrial health and established two quantitative measurements (mitochondrial fragmentation as measured by aspect ratio, and MMP as measured by the JC-10 assay) of mitochondrial defects in RTT cells. Being an essential organelle for all eukaryotic cells, mitochondrion produces energy, buffers calcium, and regulates the generation of reactive oxygen species. A better understanding of the mechanism underlying mitochondrial dysfunction in RTT is critical for the identification of drug targets and the development of effective therapies. In that regard, our work not only provides a foundation for further investigating the molecular basis of mitochondrial defects in RTT but also identifies a robust disease phenotype (reduced MMP) and a convenient assay (the JC-10 assay) that can be used to screen candidate drugs in the future.

Previous mouse studies have shown that CREB regulates MeCP2 level through microRNA (Klein et al., 2007), associates with MeCP2 to activate gene transcription (Chahrour et al., 2008), and is bidirectionally regulated by MeCP2 (Cheng et al., 2014). Our study substantially extends these previous reports by providing the first functional evidence that reduced CREB expression is responsible for key RTT pathologies at the cellular level. Specifically, we showed that overexpression of CREB in $M E C P 2$ mutant neurons is sufficient to rescue the phenotypes of reduced neuronal growth, mitochondrial fragmentation, and lower MMP. In addition to molecular manipulation, treatment with rolipram, which indirectly activates CREB signaling, can also rescue the same set of cellular RTT pathologies in MECP2 mutant neurons. Along with our finding that CREB level is reduced in MECP2 mutant neurons, these results strongly argue that CREB signaling acts downstream of MECP2 to mediate RTT pathogenesis. Furthermore, our work is done in human neurons.

In acute brain slices prepared from the $M e c p 2^{-/ y}$ mice, rolipram has been shown to rescue the LTP deficit (Balakrishnan et al.,
2016). We independently decided to test rolipram following our findings on the important role of CREB in underlying cellular RTT pathologies in human MECP2 mutant neurons. By showing that chronic treatment with rolipram can alleviate some behavioral phenotypes in the female $M e c p 2^{-/+}$mice, we illustrate the potential of CREB signaling as a molecular target for treating RTT. Although our data suggest that rolipram treatment can increase the level of pCREB, an indication of CREB activation, we recognize that the direct pharmacological effect of rolipram is inhibition of PDE-4 and increased cAMP level. Since a previous study reported that cAMP homeostasis is altered in the Mecp $2^{-/ y}$ mice (Mironov et al., 2011), it is possible that rolipram treatment acted through other signaling pathways to alleviate RTT phenotypes. Mouse genetics approaches that directly alter CREB level in vivo should help to distinguish these possibilities.

In cells, cAMP level is directly regulated by the activity of adenylate cyclase (generating cAMP) and phosphodiesterase (PDE, removing CAMP). Among all the PDE family members, PDE-4 is the most abundant in the mammalian brain. Rolipram is a first generation PDE-4 inhibitor known to increase cAMP level and subsequently activate CREB. We chose to first evaluate rolipram in our study because it was well tolerated by rodents and effective in rodent models of several neurological diseases (Gong et al., 2004; Vecsey et al., 2009; Titus et al., 2013; Choi et al., 2015; Myeku et al., 2016). However, rolipram has a short half-life in vivo and causes significant gastrointestinal side effects. Therefore, rolipram may not be suitable as a long-term treatment for RTT. It will be interesting to test other PDE-4 inhibitors in RTT mice in the future. Such candidates include PF-06266047, a PDE-4 inhibitor with superior blood-brain barrier permeability and better pharmacokinetics, and roflumilast, an FDA-approved drug used to reduce the risk of exacerbations in patients with chronic obstructive pulmonary disease (Hatzelmann et al., 2010; Rabe, 2011).

As the most recognizable RTT phenotype at the cellular level, reduced neuronal growth is not well understood at the molecular level. Previous studies are limited to studying the role of neurotrophic and growth factors in underlying this phenotype (Chang et al., 2006; Tropea et al., 2009; Castro et al., 2014). Our work revealed CREB as the downstream effector of MECP2 and WNT2 as the downstream effector of CREB to mediate the neuronal growth phenotype in $M E C P 2$ mutant neurons. By adding transcription factor and signaling molecules to the mix, our study not only advances the molecular understanding of this hallmark RTT pathology, but also identifies potential drug targets for treating it.

In conclusion, our study establishes a robust human stem cell-based platform for studying the cellular and molecular mechanisms underlying RTT pathogenesis, reveals the role of reduced CREB expression in RTT pathogenesis both in vitro and in vivo, and predicts enhancing CREB signaling as a potential treatment for RTT.

\section{References}

Amir RE, Van den Veyver IB, Wan M, Tran CQ, Francke U, Zoghbi HY (1999) Rett syndrome is caused by mutations in X-linked MECP2, encoding methyl-CpG-binding protein 2. Nat Genet 23:185-188. CrossRef Medline

Ananiev G, Williams EC, Li H, Chang Q (2011) Isogenic pairs of wild type and mutant induced pluripotent stem cell (iPSC) lines from Rett syndrome patients as in vitro disease model. PLoS One 6:e25255. CrossRef Medline

Armstrong D, Dunn JK, Antalffy B, Trivedi R (1995) Selective dendritic 
alterations in the cortex of Rett syndrome. J Neuropathol Exp Neurol 54:195-201. CrossRef Medline

Balakrishnan S, Niebert M, Richter DW (2016) Rescue of cyclic AMP mediated long term potentiation impairment in the hippocampus of Mecp2 knockout (Mecp2(-/y)) mice by rolipram. Front Cell Neurosci 10:15. CrossRef Medline

Belichenko NP, Belichenko PV, Li HH, Mobley WC, Francke U (2008) Comparative study of brain morphology in Mecp2 mutant mouse models of Rett syndrome. J Comp Neurol 508:184-195. CrossRef Medline

Belichenko PV, Wright EE, Belichenko NP, Masliah E, Li HH, Mobley WC, Francke U (2009) Widespread changes in dendritic and axonal morphology in Mecp2-mutant mouse models of Rett syndrome: evidence for disruption of neuronal networks. J Comp Neurol 514:240-258. CrossRef Medline

Brown K, Selfridge J, Lagger S, Connelly J, De Sousa D, Kerr A, Webb S, Guy J, Merusi C, Koerner MV, Bird A (2016) The molecular basis of variable phenotypic severity among common missense mutations causing Rett syndrome. Hum Mol Genet 25:558-570. CrossRef Medline

Castro J, Garcia RI, Kwok S, Banerjee A, Petravicz J, Woodson J, Mellios N, Tropea D, Sur M (2014) Functional recovery with recombinant human IGF1 treatment in a mouse model of Rett Syndrome. Proc Natl Acad Sci U S A 111:9941-9946. CrossRef Medline

Chahrour M, Jung SY, Shaw C, Zhou X, Wong ST, Qin J, Zoghbi HY (2008) $\mathrm{MeCP} 2$, a key contributor to neurological disease, activates and represses transcription. Science 320:1224-1229. CrossRef Medline

Chambers SM, Fasano CA, Papapetrou EP, Tomishima M, Sadelain M, Studer L (2009) Highly efficient neural conversion of human ES and iPS cells by dual inhibition of SMAD signaling. Nat Biotechnol 27:275-280. CrossRef Medline

Chang Q, Khare G, Dani V, Nelson S, Jaenisch R (2006) The disease progression of Mecp2 mutant mice is affected by the level of BDNF expression. Neuron 49:341-348. CrossRef Medline

Chen RZ, Akbarian S, Tudor M, Jaenisch R (2001) Deficiency of methylCpG binding protein-2 in CNS neurons results in a Rett-like phenotype in mice. Nat Genet 27:327-331. CrossRef Medline

Chen Y, Cao J, Xiong M, Petersen AJ, Dong Y, Tao Y, Huang CT, Du Z, Zhang SC (2015) Engineering human stem cell lines with inducible gene knockout using CRISPR/Cas9. Cell Stem Cell 17:233-244. CrossRef Medline

Cheng TL, Wang Z, Liao Q, Zhu Y, Zhou WH, Xu W, Qiu Z (2014) MeCP2 suppresses nuclear microRNA processing and dendritic growth by regulating the DGCR8/Drosha complex. Dev Cell 28:547560. CrossRef Medline

Cheung AY, Horvath LM, Grafodatskaya D, Pasceri P, Weksberg R, Hotta A, Carrel L, Ellis J (2011) Isolation of MECP2-null Rett Syndrome patient hiPS cells and isogenic controls through X-chromosome inactivation. Hum Mol Genet 20:2013-2115. CrossRef Medline

Choi CH, Schoenfeld BP, Weisz ED, Bell AJ, Chambers DB, Hinchey J, Choi RJ, Hinchey P, Kollaros M, Gertner MJ, Ferrick NJ, Terlizzi AM, Yohn N, Koenigsberg E, Liebelt DA, Zukin RS, Woo NH, Tranfaglia MR, Louneva $\mathrm{N}$, Arnold SE, et al. (2015) PDE-4 inhibition rescues aberrant synaptic plasticity in Drosophila and mouse models of fragile X syndrome. J Neurosci 35:396-408. CrossRef Medline

Coker SB, Melnyk AR (1991) Rett syndrome and mitochondrial enzyme deficiencies. J Child Neurol 6:164-166. CrossRef Medline

Cong L, Ran FA, Cox D, Lin S, Barretto R, Habib N, Hsu PD, Wu X, Jiang W, Marraffini LA, Zhang F (2013) Multiplex genome engineering using CRISPR/Cas systems. Science 339:819-823. CrossRef Medline

Cornford ME, Philippart M, Jacobs B, Scheibel AB, Vinters HV (1994) Neuropathology of Rett syndrome: case report with neuronal and mitochondrial abnormalities in the brain. J Child Neurol 9:424-431. CrossRef Medline

Dagda RK, Cherra SJ 3rd, Kulich SM, Tandon A, Park D, Chu CT (2009) Loss of PINK1 function promotes mitophagy through effects on oxidative stress and mitochondrial fission. J Biol Chem 284:13843-13855. CrossRef Medline

Dotti MT, Manneschi L, Malandrini A, De Stefano N, Caznerale F, Federico A (1993) Mitochondrial dysfunction in Rett syndrome: an ultrastructural and biochemical study. Brain Dev 15:103-106. CrossRef Medline

Du ZW, Chen H, Liu H, Lu J, Qian K, Huang CL, Zhong X, Fan F, Zhang SC (2015) Generation and expansion of highly pure motor neuron progen- itors from human pluripotent stem cells. Nat Commun 6:6626. CrossRef Medline

Eeg-Olofsson O, al-Zuhair AG, Teebi AS, Daoud AS, Zaki M, Besisso MS, Al-Essa MM (1990) Rett syndrome: a mitochondrial disease? J Child Neurol 5:210-214. CrossRef Medline

Gascón S, Paez-Gomez JA, Díaz-Guerra M, Scheiffele P, Scholl FG (2008) Dual-promoter lentiviral vectors for constitutive and regulated gene expression in neurons. J Neurosci Methods 168:104-112. CrossRef Medline

Goffin D, Allen M, Zhang L, Amorim M, Wang IT, Reyes AR, MercadoBerton A, Ong C, Cohen S, Hu L, Blendy JA, Carlson GC, Siegel SJ, Greenberg ME, Zhou Z (2011) Rett syndrome mutation MeCP2 T158A disrupts DNA binding, protein stability and ERP responses. Nat Neurosci 15:274-283. CrossRef Medline

Gold WA, Williamson SL, Kaur S, Hargreaves IP, Land JM, Pelka GJ, Tam PP, Christodoulou J (2014) Mitochondrial dysfunction in the skeletal muscle of a mouse model of Rett syndrome (RTT): implications for the disease phenotype. Mitochondrion 15:10-17. CrossRef Medline

Gong B, Vitolo OV, Trinchese F, Liu S, Shelanski M, Arancio O (2004) Persistent improvement in synaptic and cognitive functions in an Alzheimer mouse model after rolipram treatment. J Clin Invest 114:1624-1634. CrossRef Medline

Grosser E, Hirt U, Janc OA, Menzfeld C, Fischer M, Kempkes B, Vogelgesang S, Manzke TU, Opitz L, Salinas-Riester G, Müller M (2012) Oxidative burden and mitochondrial dysfunction in a mouse model of Rett syndrome. Neurobiol Dis 48:102-114. CrossRef Medline

Guy J, Hendrich B, Holmes M, Martin JE, Bird A (2001) A mouse Mecp2null mutation causes neurological symptoms that mimic Rett syndrome. Nat Genet 27:322-326. CrossRef Medline

Hagberg B (1985) Rett's syndrome: prevalence and impact on progressive severe mental retardation in girls. Acta Paediatr Scand 74:405-408. CrossRef Medline

Hatzelmann A, Morcillo EJ, Lungarella G, Adnot S, Sanjar S, Beume R, Schudt C, Tenor H (2010) The preclinical pharmacology of roflumilast: a selective, oral phosphodiesterase 4 inhibitor in development for chronic obstructive pulmonary disease. Pulm Pharmacol Ther 23:235-256. CrossRef Medline

Hsu PD, Scott DA, Weinstein JA, Ran FA, Konermann S, Agarwala V, Li Y, Fine EJ, Wu X, Shalem O, Cradick TJ, Marraffini LA, Bao G, Zhang F (2013) DNA targeting specificity of RNA-guided Cas9 nucleases. Nat Biotechnol 31:827-832. CrossRef Medline

Jan YN, Jan LY (2010) Branching out: mechanisms of dendritic arborization. Nat Rev Neurosci 11:316-328. CrossRef Medline

Jugloff DG, Jung BP, Purushotham D, Logan R, Eubanks JH (2005) Increased dendritic complexity and axonal length in cultured mouse cortical neurons overexpressing methyl-CpG-binding protein MeCP2. Neurobiol Dis 19:18-27. CrossRef Medline

Kim KY, Hysolli E, Park IH (2011) Neuronal maturation defect in induced pluripotent stem cells from patients with Rett syndrome. Proc Natl Acad Sci U S A 108:13169-14174. CrossRef Medline

Kishi N, Macklis JD (2004) MECP2 is progressively expressed in postmigratory neurons and is involved in neuronal maturation rather than cell fate decisions. Mol Cell Neurosci 27:306-321. CrossRef Medline

Klein ME, Lioy DT, Ma L, Impey S, Mandel G, Goodman RH (2007) Homeostatic regulation of MeCP2 expression by a CREB-induced microRNA. Nat Neurosci 10:1513-1514. CrossRef Medline

Lewis JD, Meehan RR, Henzel WJ, Maurer-Fogy I, Jeppesen P, Klein F, Bird A (1992) Purification, sequence, and cellular localization of a novel chromosomal protein that binds to methylated DNA. Cell 69:905-914. CrossRef Medline

Li H, Zhong X, Chau KF, Williams EC, Chang Q (2011) Loss of activityinduced phosphorylation of MeCP2 enhances synaptogenesis, LTP and spatial memory. Nat Neurosci 14:1001-1008. CrossRef Medline

Li YF, Huang Y, Amsdell SL, Xiao L, O’Donnell JM, Zhang HT (2009) Antidepressant- and anxiolytic-like effects of the phosphodiesterase- 4 inhibitor rolipram on behavior depend on cyclic AMP response element binding protein-mediated neurogenesis in the hippocampus. Neuropsychopharmacology 34:2404-2419. CrossRef Medline

Li Y, Wang H, Muffat J, Cheng AW, Orlando DA, Lovén J, Kwok SM, Feldman DA, Bateup HS, Gao Q, Hockemeyer D, Mitalipova M, Lewis CA, Vander Heiden MG, Sur M, Young RA, Jaenisch R (2013) Global transcriptional and translational repression in human-embryonic-stem-cell- 
derived Rett syndrome neurons. Cell Stem Cell 13:446-458. CrossRef Medline

Mali P, Yang L, Esvelt KM, Aach J, Guell M, DiCarlo JE, Norville JE, Church GM (2013) RNA-guided human genome engineering via Cas9. Science 339:823-826. CrossRef Medline

Marchetto MC, Carromeu C, Acab A, Yu D, Yeo GW, Mu Y, Chen G, Gage FH, Muotri AR (2010) A model for neural development and treatment of Rett syndrome using human induced pluripotent stem cells. Cell 143: 527-539. CrossRef Medline

Merrill RA, Dagda RK, Dickey AS, Cribbs JT, Green SH, Usachev YM, Strack S (2011) Mechanism of neuroprotective mitochondrial remodeling by PKA/AKAP1. PLoS Biol 9:e1000612. CrossRef Medline

Mironov SL, Skorova EY, Kügler S (2011) Epac-mediated cAMP-signalling in the mouse model of Rett Syndrome. Neuropharmacology 60:869-877. CrossRef Medline

Myeku N, Clelland CL, Emrani S, Kukushkin NV, Yu WH, Goldberg AL, Duff KE (2016) Tau-driven $26 \mathrm{~S}$ proteasome impairment and cognitive dysfunction can be prevented early in disease by activating cAMP-PKA signaling. Nat Med 22:46-53. CrossRef Medline

Nan X, Campoy FJ, Bird A (1997) MeCP2 is a transcriptional repressor with abundant binding sites in genomic chromatin. Cell 88:471-481. CrossRef Medline

Pitcher MR, Herrera JA, Buffington SA, Kochukov MY, Merritt JK, Fisher AR, Schanen NC, Costa-Mattioli M, Neul JL (2015) Rett syndrome like phenotypes in the R255X Mecp2 mutant mouse are rescued by MECP2 transgene. Hum Mol Genet 24:2662-2672. CrossRef Medline

Rabe KF (2011) Update on roflumilast, a phosphodiesterase 4 inhibitor for the treatment of chronic obstructive pulmonary disease. Br J Pharmacol 163:53-67. CrossRef Medline

Redmond L, Kashani AH, Ghosh A (2002) Calcium regulation of dendritic growth via CaM kinase IV and CREB-mediated transcription. Neuron 34:999-1010. CrossRef Medline

Ryu H, Lee J, Impey S, Ratan RR, Ferrante RJ (2005) Antioxidants modulate mitochondrial PKA and increase CREB binding to D-loop DNA of the mitochondrial genome in neurons. Proc Natl Acad Sci U S A 102:1391513920. CrossRef Medline

Samaco RC, Neul JL (2011) Complexities of Rett Syndrome and MeCP2. J Neurosci 31:7951-7959. CrossRef Medline

Samaco RC, McGraw CM, Ward CS, Sun Y, Neul JL, Zoghbi HY (2013)
Female Mecp2(+/-) mice display robust behavioral deficits on two different genetic backgrounds providing a framework for pre-clinical studies. Hum Mol Genet 22:96-109. CrossRef Medline

Schaevitz LR, Gómez NB, Zhen DP, Berger-Sweeney JE (2013) MeCP2 R168X male and female mutant mice exhibit Rett-like behavioral deficits. Genes Brain Behav 12:732-740. CrossRef Medline

Shahbazian M, Young J, Yuva-Paylor L, Spencer C, Antalffy B, Noebels J, Armstrong D, Paylor R, Zoghbi H (2002) Mice with truncated MeCP2 recapitulate many Rett syndrome features and display hyperacetylation of histone H3. Neuron 35:243-254. CrossRef Medline

Soldner F, Jaenisch R (2012) Medicine: iPSC disease modeling. Science 338: 1155-1156. CrossRef Medline

Titus DJ, Sakurai A, Kang Y, Furones C, Jergova S, Santos R, Sick TJ, Atkins CM (2013) Phosphodiesterase inhibition rescues chronic cognitive deficits induced by traumatic brain injury. J Neurosci 33:5216-5226. CrossRef Medline

Tropea D, Giacometti E, Wilson NR, Beard C, McCurry C, Fu DD, Flannery R, Jaenisch R, Sur M (2009) Partial reversal of Rett Syndrome-like symptoms in MeCP2 mutant mice. Proc Natl Acad Sci U S A 106:2029_ 2034. CrossRef Medline

Vecsey CG, Baillie GS, Jaganath D, Havekes R, Daniels A, Wimmer M, Huang T, Brown KM, Li XY, Descalzi G, Kim SS, Chen T, Shang YZ, Zhuo M, Houslay MD, Abel T (2009) Sleep deprivation impairs cAMP signalling in the hippocampus. Nature 461:1122-1125. CrossRef Medline

Wayman GA, Impey S, Marks D, Saneyoshi T, Grant WF, Derkach V, Soderling TR (2006) Activity-dependent dendritic arborization mediated by CaM-kinase I activation and enhanced CREB-dependent transcription of Wnt-2. Neuron 50:897-909. CrossRef Medline

Wegener E, Brendel C, Fischer A, Hülsmann S, Gärtner J, Huppke P (2014) Characterization of the MeCP2R168X knockin mouse model for Rett syndrome. PLoS One 9:e115444. CrossRef Medline

Williams EC, Zhong X, Mohamed A, Li R, Liu Y, Dong Q, Ananiev GE, Mok JC, Lin BR, Lu J, Chiao C, Cherney R, Li H, Zhang SC, Chang Q (2014) Mutant astrocytes differentiated from Rett syndrome patients-specific iPSCs have adverse effects on wild-type neurons. Hum Mol Genet 23: 2968-2980. CrossRef Medline

Zhong X, Li H, Chang Q (2012) MeCP2 phosphorylation is required for modulating synaptic scaling through mGluR5. J Neurosci 32:1284112847. CrossRef Medline 